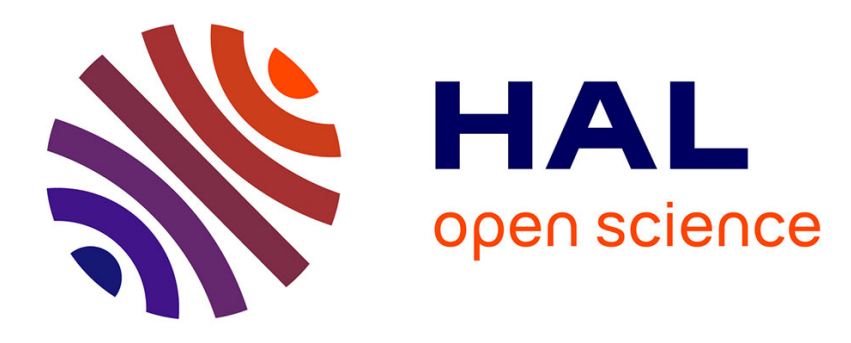

\title{
Le capital marque: proposition d'une mesure individuelle et essai de validation
}

Philippe Jourdan

\section{To cite this version:}

Philippe Jourdan. Le capital marque: proposition d'une mesure individuelle et essai de validation.

Recherche et Applications en Marketing (French Edition), 2001, 16, pp.3-23. hal-01132968

\section{HAL Id: hal-01132968 \\ https://hal.science/hal-01132968}

Submitted on 18 Mar 2015

HAL is a multi-disciplinary open access archive for the deposit and dissemination of scientific research documents, whether they are published or not. The documents may come from teaching and research institutions in France or abroad, or from public or private research centers.
L'archive ouverte pluridisciplinaire HAL, est destinée au dépôt et à la diffusion de documents scientifiques de niveau recherche, publiés ou non, émanant des établissements d'enseignement et de recherche français ou étrangers, des laboratoires publics ou privés. 


\title{
Le capital marque : proposition d'une mesure individuelle et essai de validation
}

Philippe JOURDAN

Maître de Conférences

IUT d'Evry Val Essonne

Directeur Général JPL Consulting

\author{
ADRESSE PROFESSIONNELLE : \\ CERAG \\ Ecole Supérieure des Affaires (ESA) \\ Université Pierre Mendés France \\ Boite postale 47 \\ 38040 Grenoble Cedex 9
}

\author{
ADRESSE PERSONNELLE : \\ 26, rue Richer \\ 75009 Paris \\ Téléphone : $33(0) 148008144$ \\ Télécopie : 33 (0)148008144 \\ Mél. : Philippe.jourdan@panelontheweb.com
}

\section{REMERCIEMENTS :}

L'auteur remercie les sociétés GfK Marketing Services et IRI-Secodip en la personne de monsieur Gérard HERMET, membre du directoire de GfK A.G., et de messieurs JeanBernard KAZMIERCZAK et Alain QUAGHEBEUR, directeurs scientifiques de IRI et de SECODIP, pour l'apport des données panels utilisées dans cette article. Sincères remerciements à messieurs Alain JOLIBERT, Chan SU PARK et V. Seenu SRINIVASAN pour la pertinence de leurs remarques et de leurs commentaires ainsi qu'à Valérie pour les soins apportés à la relecture de cet article. 


\title{
Le capital marque : proposition d'une mesure individuelle et essai de validation
}

\begin{abstract}
RESUME :
Cet article introduit une méthode de mesure individuelle du capital marque fondée sur les préférences et les choix du consommateur. Prenant appui sur les travaux de Park et Srinivasan, l'auteur propose deux améliorations, l'une portant sur le cadre conceptuel, l'autre sur la méthode de mesure employée. Une expérimentation, confrontant des données de laboratoire et de panels sur 32 marques différentes, indique une meilleure validité interne et externe de la méthode révisée. Le construit de capital marque corrigé se révèle effectivement plus fortement corrélé à d'autres indicateurs de la force de la marque, tels que la part de marché, la distribution numérique et la distribution valeur. Enfin, le modèle de prévision, élaboré à partir du construit, conduit à une bonne estimation des parts de choix, témoignant de la validité prédictive de la méthode. Certaines limites qui forment autant de pistes de recherche sont soulignées : l'article se positionne à un stade exploratoire et certains des résultats rapportés nécessitent un approfondissement confirmatoire.
\end{abstract}

Mots clés: analyse conjointe - capital marque - marque - biens durables - biens de grande consommation - panel de détaillants - parts de marché

\section{Brand Equity: Proposal for an Individual Measurement and Attempt at Validation}

\begin{abstract}
:
The purpose of this article is to come up with an individual measure of brand equity, based on consumers preferences and choices. Using the customer-based brand equity definition and valuation method, initially proposed by Park and Srinivasan, the authors put forwards two improvements, one conceptual, the other methodological. A survey-based results on 32 brands, comparing laboratory data and panel data, shows that the revised method has a better internal and external validity. The corrected brand equity construct is indeed better correlated to other constructs related to brand strength, such as market share and distribution indices. Last but not least, the Logit model, elaborated upon the revised construct, leads to a satisfactory prediction of choice shares, attesting that the proposed approach has a good predictive validity. Some limits which are paths for new research are underlined : the article is still exploratory and some of the results call for a confirmatory validation.
\end{abstract}

Keywords : conjoint analysis - brand equity - brand - durable goods - fast moving consumer goods retail panel - market share 


\section{Le capital marque : proposition d'une mesure individuelle et essai de validation}

\section{1)- INTRODUCTION}

La marque peut être utilement définie comme un «attribut visible, physique et durable, apposé sur un corps pour le distinguer» (Lewi, 1996). Autrefois simple support de l'identité du fabricant, la marque exerce, de nos jours, un rôle de premier plan : il serait actuellement impensable de lancer un nouveau produit, sans lui attribuer un nom et surtout sans le doter d'une personnalité. Toutefois, la mise au point de méthodes de mesure du rôle de la marque (appelées mesures du capital marque) est une préoccupation récente de la recherche en marketing (Marketing Science Institute, 1988). Parmi les différentes méthodes éprouvées, celles qui s'appuient sur le point de vue du consommateur occupent une place éminente (Farquhar, 1989 ; Aaker, 1992 ; Barwise, 1993 ; Kamakura et Russell, 1993 ; Keller, 1993 ; Park et Srinivasan, 1994). Notre recherche tente d'améliorer l'une d'entre elle, celle mise au point par Park (1992) et Park et Srinivasan (1994). Cette amélioration dans la définition des construits et la précision de la mesure - que nous situons à un stade encore exploratoire permet d'aboutir à une mesure individuelle du capital marque. Une première validation est ensuite effectuée : les résultats sont présentés et les limites soulignées.

Dans un premier temps, nous rappelons le champ théorique, dans lequel s'inscrit le raisonnement de Park et Srinivasan. Puis, nous présentons les différences entre notre approche et celle des auteurs. Enfin, nous comparons les résultats produits par chacune des méthodes au moyen d'une expérimentation, afin de vérifier le bien-fondé des améliorations que nous proposons.

\section{2)- LES APPROCHES CENTREES SUR LE POINT DE VUE DU CONSOMMATEUR}

Jusqu'à la fin des années 70, les chercheurs se sont le plus souvent intéressés à l'ensemble que forment le produit et sa marque, n'établissant aucune distinction entre l'impact de la marque et celui du produit. L'article de Srinivasan (1979), qui démontre que la marque dispose d'une valeur (utilité) propre, indépendante de celle du produit, et les travaux du Marketing Science Institute (Leuthesser, 1988) remettent radicalement en cause cette conception : la marque et le produit forment en réalité deux composantes distinctes de l'offre. Cette séparation est à l'origine du concept de capital marque, qu'un groupe d'experts, réunis par le MSI (1988), définit comme «l'ensemble des associations et des comportements (...) qui permettent aux produits marqués de réaliser des volumes de ventes et des marges plus importants que ceux qu'ils pourraient atteindre sans la mention de la marque ». Depuis lors, la recherche en matière de valorisation de la marque se développe autour de deux approches (Srivastava et Shocker, 1991). La première regroupe l'ensemble des méthodes comptables et financières de valorisation du portefeuille des marques de l'entreprise («Firm-Based Brand Equity »). La deuxième, que nous retenons, postule que la valeur de la marque repose sur 
l'influence qu'elle exerce sur le choix et la décision d'achat d'un produit par le consommateur ( Customer-Based Brand Equity »).

Le capital marque a la particularité d'être un construit qui n'est pas directement observable : il n'est donc mesurable qu'à travers ses manifestations. De ce fait, les chercheurs recourent à deux méthodes. La première méthode privilégie deux dimensions perceptives : l'attention portée à la marque, que mesurent les indicateurs de notoriété spontanée et assistée (Nedungadi, 1990 ; Alba et al., 1991) et l'évaluation de son image (Shocker et Weitz, 1988 ; Biel, 1992 ; Krishnan, 1996), qui se matérialise dans la force, la valence, l'unicité, et plus accessoirement la cohérence et la pertinence des associations qu'éveille la marque dans l'esprit du consommateur (Keller, 1993). La deuxième méthode utilise la préférence du consommateur. Dans ce contexte, l'analyse des mesures conjointes permet de dissocier la performance de la marque de celle des autres attributs du produit et d'isoler ainsi la contribution de la marque à la formation d'une préférence globale, traduite sous la forme d'une utilité ${ }^{1}$.

\section{A)- LES MÉTHODES DE MESURE DIRECTE DU CAPITAL MARQUE}

Sont ainsi désignées les méthodes de valorisation du capital marque fondées sur la mesure directe de la préférence ou des choix du consommateur. Cette mesure s'effectue soit par régression Logit (Swait et al., 1993 ; Kamakura et Russell, 1993), soit par l'analyse conjointe (Srinivasan, 1979; Park et Srinivasan, 1994). En réalité, la justification théorique de ces deux approches repose sur le modèle du comportement du consommateur d'Urban et Hauser (1980). Les deux auteurs postulent que le consommateur évalue le produit à partir de ses caractéristiques et d'après les sources d'information externes (publicité, bouche à oreille, etc.). Parce que le consommateur cherche à maximiser sa fonction d'utilité, la détermination d'une préférence repose à la fois sur l'évaluation du produit et sur les motivations individuelles d'achat. Enfin, le choix est fonction de la préférence du consommateur que modèrent toutefois des variables externes, telles que le budget alloué à l'achat, le prix du produit ou bien sa disponibilité en magasin.

Il découle de ce modèle que la marque, considérée comme un attribut extrinsèque du produit, exerce un impact aussi bien sur l'évaluation de ses caractéristiques objectives que sur la préférence et le choix. Le capital marque peut donc être assimilé à la part d'utilité résiduelle que n'explique pas l'évaluation objective des attributs du produit (Shocker et Weitz, 1988 ; Swait et al. 1993). De façon plus opérationnelle, Park et Srinivasan (1994) mesurent le capital marque comme la différence entre la préférence globale vis-à-vis du "produit marqué » et une valeur d'utilité fondée sur l'évaluation objective des seuls attributs du produit. Cette dernière approche fait précisément l'objet de notre article.

Les méthodes de valorisation du capital de la marque qui recourent à l'analyse des mesures conjointes présentent trois qualités. Sous certaines conditions, elles permettent

La littérature anglo-saxonne sur l'analyse conjointe utilise indifféremment le mot « utilité » et « préférence ». Toutefois, selon les échelles d'interrogation choisie, l'utilité - qui désigne l'indicateur de mesure - peut être différente d'une mesure de la préférence. Ainsi on peut préférer une marque tout en jugeant plus « utile » l'achat d'une autre marque. Par la suite et compte-tenu des échelles de mesure utilisées, nous adoptons indifféremment les termes utilités et préférences pour désigner un même concept : l'expression d'un choix indépendamment de toute contrainte externe. 
d'obtenir une mesure individuelle du capital marque, et non une mesure agrégée au niveau du marché ou de certains segments (Kamakura et Russell, 1993). Elles dissocient clairement l'utilité qui s'attache au produit de celle qu'explique la marque (Park et Srinivasan, 1994). Enfin, leur application conduit à isoler l'impact de la marque, selon qu'il s'exerce sur l'évaluation des caractéristiques du produit (effet de halo ou d'inférence) ou sur la préférence globale (effet d'heuristique).

\section{B)- LA VALORISATION DE L'IMPACT DE LA MARQUE}

A première vue, l'utilisation de l'analyse conjointe pour mesurer la valeur du capital marque semble être la solution la plus appropriée (Green et Srinivasan, 1990). Toutefois, assimiler la marque à un attribut du produit au même titre que ses autres caractéristiques soulève deux problèmes. La mesure statistique des effets d'interaction entre la marque et les autres attributs est complexe (Rangaswamy et al., 1993). Enfin, parce que le consommateur associe la marque à des caractéristiques précises, la manipulation de cette variable dans des plans expérimentaux aboutit à des scénarii parfois irréalistes, ce qui entache la validité externe de l'expérimentation (Green et Srinivasan, 1978).

\section{L'IMPACT GLOBAL DE LA MARQUE}

Pour pallier cette dernière difficulté, Srinivasan (1979) conseille de ne pas introduire la marque comme variable dans le plan d'expérience. L'auteur considère que son impact doit être assimilé à "la part de préférence globale que n'explique pas le modèle multiattribut ». Dès lors, la préférence globale de l'individu i pour la marque $\mathrm{j}\left(\mathrm{B}{ }_{\mathrm{ij}}{ }_{\mathrm{j}}\right)$ est égale à la préférence calculée à l'aide d'un modèle multiattribut compensatoire $\left(\mathrm{B}_{\mathrm{ij}}\right)$, auquel s'ajoute une contribution spécifique imputable à la marque $\left(\mathrm{V}_{\mathrm{ij}}\right)$. Srinivasan démontre que la prise en compte de cet effet, dû à la marque, améliore notablement le pouvoir prédictif du modèle d'analyse conjointe.

Cette formulation remaniée de la préférence globale est séduisante. La méthodologie employée appelle toutefois trois remarques. En premier lieu, Srinivasan (1979) est contraint de considérer que la valeur de la part de préférence qu'explique la marque ne dépend pas des sujets interrogés, pour éviter que ce construit ne devienne tautologique. En effet, parce que l'effet propre à la marque $\left(\mathrm{V}_{\mathrm{ij}}\right)$ est valorisé par différence, on peut toujours prédire la préférence de n'importe quel individu $\left(\mathrm{B}^{\prime}{ }_{\mathrm{ij}}\right)$ en assignant à chaque sujet une valeur spécifiquement calculée à $V_{i j}$ ! Si elle se justifie sur un plan méthodologique, la démarche de calcul de l'auteur s'oppose au principe d'une mesure individuelle du capital de la marque. Deuxième remarque : l'effet propre de la marque $\left(\mathrm{V}_{\mathrm{ij}}\right)$, considéré comme unidimensionnel, agrège en réalité deux influences distinctes, l'impact sur l'évaluation des attributs et celui sur la préférence globale (Park, 1992). Enfin, le construit $V_{i j}$ ne valorise pas uniquement l'impact de la marque : par sa formulation, il englobe aussi l'erreur de mesure, sans qu'il soit possible de la dissocier, imputable aussi bien à l'utilisation d'un modèle multiattribut particulier - en l'occurrence, le modèle linéaire additif - qu'à l'arbitraire du choix des critères d'évaluation du produit (Mazis et al., 1975). En effet, la validité de la méthode d'analyse des mesures conjointes est dépendante de la sélection des attributs et de leur caractère déterminant dans le processus de choix du consommateur (Green et 
Srinivasan, 1978), vérifications que nous effectuons préalablement à notre expérimentation.

\section{LA DECOMPOSITION EN DEUX DIMENSIONS DE L'IMPACT DE LA MARQUE}

Bien que formulée d'une façon légèrement différente, la définition du capital marque adoptée par Park (1992) est finalement très proche de celle énoncée par Srinivasan (1979). Park valorise, à son tour, le capital marque en procédant au calcul de «la différence entre la préférence globale pour le produit marqué et la préférence fondée sur les attributs du produit et calculée à l'aide d'un modèle multiattribut ». L'originalité de la modélisation, proposée ensuite par Park et Srinivasan (1994), réside dans la décomposition du capital marque global en deux composantes, notées respectivement $\mathrm{a}_{\mathrm{ij}}$ et $\mathrm{n}_{\mathrm{ij}}$. La première $\left(\mathrm{a}_{\mathrm{ij}}\right)$, communément dénommée " effet de halo » (Wilkie et Pessemier, 1973 ; Holbrook et Huber, 1979 ; Alba et Hutchinson, 1987 ), mesure la distorsion due à la marque sur l'évaluation des caractéristiques du produit. La seconde composante $\left(\mathrm{n}_{\mathrm{ij}}\right)$ capture l'effet résiduel de la marque sur la préférence globale, plus couramment appelé " effet d'heuristique » (Wyer et Srull, 1986; Alba et Marmorstein, 1987). Lors du recours à une heuristique de jugement, le consommateur s'appuie sur la marque, qu'il considère comme un critère d'ancrage et d'ajustement (Tversky et Kahneman, 1974), pour estimer globalement la qualité du produit sans pour autant évaluer de manière détaillée ses caractéristiques. Un tel processus corrobore les conclusions de Hirschman et Holbrook (1982) et de Zajonc et Marcus (1982), qui soulignent la défaillance des modèles multiattributs à prédire correctement le choix des consommateurs s'agissant des biens de luxe et des produits à forte connotation d'image. Ce faisant, Park et Srinivasan formulent l'équation fondamentale suivante :

(1) $\mathrm{e}_{\mathrm{ij}}=\mathrm{a}_{\mathrm{ij}}+\mathrm{n}_{\mathrm{ij}}$

$\mathrm{e}_{\mathrm{ij}}$ : capital marque de la marque j pour l'individu i.

$a_{\mathrm{ij}}$ : capital marque de la marque j pour l'individu i relié aux attributs du produit.

$\mathrm{n}_{\mathrm{ij}}$ : capital marque de la marque j pour l'individu i non relié aux attributs du produit.

Cette méthode a le mérite de valoriser séparément les deux contributions de la marque à la formation de l'attitude vis-à-vis du produit. A notre connaissance, elle est la seule qui mesure à la fois l'effet direct de la marque sur la préférence et son effet indirect, via l'altération de la perception des attributs du produit marqué. Afin de démontrer la robustesse et la validité de leurs construits, Park et Srinivasan se livrent à une série de vérifications. Grâce à une procédure de « test-restest», ils s'assurent de la stabilité des trois composantes du capital marque : les coefficients de corrélation entre les deux phases de test approchent ou dépassent 0,70, critère de seuil jugé satisfaisant (Nunnally, 1978). Ensuite, les auteurs vérifient la validité convergente du construit de capital marque, en confrontant sa valeur avec celle issue de l'approche de décomposition suggérée par Srinivasan (1979). Pour les produits communs aux deux études - le dentifrice et le rince-bouche -, les valeurs du capital marque sont fortement corrélées (respectivement 0,86 et 0,71$)$ : la validité convergente des construits est donc satisfaisante.

Cependant, la méthode utilisée et l'expérimentation proposées par Park et Srinivasan (1994) appellent deux réserves. D'une part, les expressions mathématiques du capital marque et de sa composante, non liée aux attributs du produit, méritent d'être 
« épurées », afin d'en soustraire un terme d'erreur. D'autre part, dans le souci d'établir une mesure individuelle du capital marque, il est préférable d'adopter un plan d'expérience à mesures répétées auprès d'un seul et même échantillon. Ces deux constats nous amènent à proposer une amélioration de la mesure individuelle du capital marque.

\section{3)- LE CAPITAL MARQUE ET SA MESURE INDIVIDUELLE}

Après avoir défini les construits que nous utilisons, nous présenterons notre méthode d'expérimentation. En effet, la mesure individuelle du capital marque que nous proposons présente deux différences avec celle de Park et Srinivasan (1994) : l'une réside dans la définition et l'expression mathématique des construits, l'autre dans le plan d'expérience mis en oeuvre.

\section{A)- DÉFINITIONS ET EXPRESSIONS MATHÉMATIQUES DES CONSTRUITS}

Rappelons que Park et Srinivasan (1994) définissent le capital marque comme «la différence entre la préférence globale du consommateur vis-à-vis de la marque et celle calculée, en s'appuyant sur une évaluation objective des attributs du produit sans la marque $»$. Il en résulte que la différence d'utilités associée à la mesure de ce construit n'est pas entièrement imputable à la marque : une partie relève de l'erreur de mesure, propre à la méthode de calcul utilisée. Deux raisons expliquent qu'en dehors de toute considération liée à la marque, la préférence qui s'appuie sur l'évaluation des attributs du produit sans la marque puisse ne pas être parfaitement corrélée à la préférence globale. Tout d'abord, le consommateur peut évaluer très favorablement tous les attributs d'un produit et lui en préférer un autre (Tversky et Kahneman, 1998), en raison de "l'incohérence» et de l'irrationalité de ses choix (Feldman et Lynch, 1988). Ce comportement se traduit par un premier type d'erreur de nature aléatoire. Ensuite, l'utilité basée sur l'évaluation objective des attributs est calculée au moyen d'un modèle multiattribut compensatoire linéaire additif : le choix arbitraire du modèle, la nature et le nombre d'attributs retenus, le non-respect de leur indépendance peuvent entraîner une erreur systématique. Ainsi s'explique l'écart qui existe lorsque la préférence est mesurée par une approche globale ou analytique (Mazis et al., 1975).

\section{B)- DÉFINITION ET MESURE DU CAPITAL MARQUE}

En considérant la marque comme une information susceptible d'altérer la préférence que le consommateur porte au produit sans la marque, nous formulons la définition suivante du capital marque :

$\mathbf{D}_{1}$ - Le capital marque est la différence entre la préférence pour le produit avec la marque et la préférence pour le produit sans la marque.

Cette définition opère une séparation plus nette entre le concept et sa mesure que celle adoptée par Park et Srinivasan (1994) : indépendamment de toute méthode expérimentale, le capital marque est assimilé à la part de préférence qui résulte de la connaissance et de l'appréciation de la marque par le consommateur. Pour calculer cette part de préférence, le consommateur sera interrogé deux fois selon une procédure 
identique : seule la variable marque est successivement "manipulée " en absence ou présence (Olson, 1977). Cette définition est différente de celle de Park et Srinivasan (1994) qui mesurent le capital marque comme la différence entre deux valeurs d'utilités calculées selon des modalités différentes. Pour faciliter la lecture et la comparaison, les conventions d'écriture suivantes seront utilisées :

$\mathrm{u}(\mathrm{s})_{\mathrm{ij}}$ : $\quad$ utilité globale lorsque l'individu i a connaissance de la marque $\mathrm{j}$.

$\mathrm{u}(\mathrm{o})_{\mathrm{ij}}$ : utilité globale lorsque la marque $\mathrm{j}$ est occultée à l'individu i.

$\mathrm{v}(\mathrm{s})_{\mathrm{ij}}$ : utilité fondée sur l'évaluation des attributs lorsque l'individu i a connaissance de la marque $\mathrm{j}$.

$\mathrm{v}(\mathrm{o})_{\mathrm{ij}}$ : utilité fondée sur l'évaluation des attributs lorsque la marque j est occultée à l'individu i.

L'expression mathématique du capital marque $\left(\mathrm{e}_{\mathrm{ij}}\right)$ qui résulte de la définition $\mathrm{D}_{1}$ est alors

$$
e_{i j}=u(s)_{i j}-u(o)_{i j}
$$

alors que Park et Srinivasan (1994) calculent la valeur de ce même construit à l'aide de l'expression :

$$
e^{\prime} i j=u(s) i j-v(o) i j
$$

Il en résulte pour tout individu i et pour toute produit $\mathrm{j}$, la différence $\xi(\mathrm{o})_{\mathrm{ij}}=\left[\mathrm{e}_{\mathrm{ij}}{ }-\mathrm{e}_{\mathrm{ij}}\right]$ :

$$
\xi(\mathrm{o}) \mathrm{ij}=(3)-(2)=\mathrm{u}(\mathrm{o}) \mathrm{ij}-\mathrm{v}(\mathrm{o}) \mathrm{ij}
$$

Cette différence est égale à la part de l'utilité (ou de la préférence) globale de l'individu $\mathrm{i}$ pour la marque $\mathrm{j}$ que n'explique pas le modèle multiattribut : elle correspond par définition au terme d'erreur décrit plus haut (cf. page 8).

\section{DEFINITION ET MESURE DU CAPITAL MARQUE FONDE SUR LES ATTRIBUTS DU PRODUIT}

Pour juger de la qualité d'un produit, le consommateur s'appuie à des degrés divers sur les attributs extrinsèques (Olson, 1972) dont la marque fait partie (Asam et Bucklin, 1973 ; Makens, 1965) pour en déduire la performance des attributs intrinsèques. Par ailleurs, la connaissance et l'image de la marque altèrent l'évaluation objective des caractéristiques du produit, distorsion plus connue sous le nom d'effet de halo (Wilkie et Pessemier, 1973 ; Alba et Hutchinson, 1987 ; Murphy et al., 1993). Ainsi se trouve justifiée la définition du capital marque fondée sur les attributs du produit, qui recoupe par ailleurs celle de Park et Srinivasan (1994) :

$\mathbf{D}_{\mathbf{2}}$ - Le capital marque fondé sur les attributs du produit est la différence entre une préférence basée sur l'évaluation des attributs du produit avec la marque et une préférence basée sur l'évaluation des attributs du produit sans la marque.

Le capital marque fondé sur les attributs du produit est mesuré par la différence des utilités obtenues par l'application d'un modèle multiattribut, selon que les attributs du produit sont évalués en présence ou en absence de la marque. Si nous désignons par $\mathrm{a}_{\mathrm{ij}}$ le capital marque fondé sur les attributs du produit, par $s_{\mathrm{ijp}}$ et $\mathrm{o}_{\mathrm{ijp}}$ l'évaluation avec la marque, puis sans la marque, de l'attribut $\mathrm{p}$ de la marque $\mathrm{j}$ par l'individu $\mathrm{i}$ et $\operatorname{par} \mathrm{v}(\mathrm{s})_{\mathrm{ij}}$ et 
$\mathrm{v}(\mathrm{o})_{\mathrm{ij}}$ l'utilité du produit avec la marque, puis sans la marque, calculée à l'aide du modèle multiattribut, nous posons alors l'équation suivante :

$$
a_{i j}=v(s)_{i j}-v(o)_{i j}=\sum_{p=1}^{q} f_{i p}\left(s_{i j p}\right)-\sum_{p=1}^{q} f_{i p}\left(o_{i j p}\right)
$$

\section{DEFINITION ET MESURE DU CAPITAL MARQUE NON FONDE SUR LES ATTRIBUTS DU PRODUIT}

L'appréciation de la valeur d'un produit repose sur de nombreuses considérations dont certaines sont étrangères à ses caractéristiques intrinsèques. En particulier, les éléments constitutifs de l'image de la marque contribuent à une partie non négligeable de la préférence globale (Keller, 1993). Cette part de préférence que ne déterminent pas les caractéristiques du produit est appelé «effet propre de la marque » (Srinivasan, 1979). Nous le dénommons « capital marque non fondé sur les attributs du produit », reprenant la terminologie employée par Park et Srinivasan (1994) et le définissons ainsi :

$\mathbf{D}_{\mathbf{3}}$ - Le capital marque non fondé sur les attributs du produit est la différence entre une préférence pour le produit avec la marque et une préférence, fondée sur l'évaluation des attributs du produit avec la marque.

Par analogie avec le raisonnement tenu pour la mesure du capital marque, nous devons soustraire du calcul de la différence entre les deux utilités la valeur du terme d'erreur individuel lié à l'application d'un modèle multiattribut. Ainsi, en reprenant les mêmes conventions d'écriture (cf. page 9), le construit de capital marque non fondé sur les attributs du produit, $\mathrm{n}_{\mathrm{ij}}$, est mesuré par l'expression mathématique suivante où $\xi(\mathrm{s})_{\mathrm{ij}}$ est le terme d'erreur aléatoire propre à 1 'individu i sur la marque $\mathrm{j}$ lorsque la marque est présentée :

$$
n_{i j}=\left[u(s)_{i j}-v(s)_{i j}\right]-\xi(s)_{i j}
$$

L'expression mathématique du construit est ici différente de celle de Park et Srinivasan (1994). Cette différence est à nouveau liée à la soustraction d'un terme d'erreur individuel $\xi(\mathrm{s})_{\mathrm{ij}}$. Puisque nous effectuons deux mesures auprès du même échantillon, nous pouvons estimer que, pour chaque sujet $\mathrm{i}$, le terme $\xi(\mathrm{s})_{\mathrm{ij}}$ calculé lorsque la marque est connue a une valeur proche de celui calculé lorsque la marque est occultée $\left(\xi(0)_{\mathrm{ij}}\right)$. En toute rigueur, $\xi(\mathrm{s})_{\mathrm{ij}}$ et $\xi(\mathrm{o})_{\mathrm{ij}}$ pourraient ne pas avoir forcément la même valeur : l'écart traduit la cohérence des réponses de l'individu, qui peut varier tout au long de l'entretien, en raison notamment de la lassitude ou de la fatigue. Toutefois, compte tenu des précautions expérimentales prises (cf. page 16), on peut raisonnablement postuler que ces deux construits sont égaux, ce qui permet d'en calculer la valeur au moyen des indices $\mathrm{u}(\mathrm{o})_{\mathrm{ij}}$ et $\mathrm{v}(\mathrm{o})_{\mathrm{ij}}$ :

$$
\xi(o)_{i j}=\xi(s)_{i j}=\left[u(o)_{i j}-v(o)_{i j}\right]
$$

\section{EQUATION FONDAMENTALE}


Le capital marque est la somme du capital marque fondé sur les attributs du produit et du capital marque non fondé sur les attributs du produit. Bien que nos définitions de deux des trois construits soient différentes de celles de Park et Srinivasan, le fait de soustraire de la valeur de ces construits le même terme d'erreur ne change pas le sens de l'égalité mais nous permet de calculer une valeur plus «juste» de deux de ses trois composantes (cf. équation 8).

$$
\begin{array}{ll}
e_{i j}=u(s)_{i j}-u(o)_{i j}=u(s)_{i j}-\left[v(o)_{i j}+\xi(o)_{i j}\right] & \text { en considérant (2) et (4) } \\
e_{i j}=\left[u(s)_{i j}-v(s)_{i j}\right]+\left[v(s)_{i j}-v(o)_{i j}\right]-\xi(o)_{i j} & \text { en rajoutant puis retranchant } v\left(s_{i j}\right. \\
e_{i j}=\left[u(s)_{i j}-v(s)_{i j}-\xi(s)_{i j}\right]+\left[v(s)_{i j}-v(o)_{i j}\right] & \text { en considérant (7) } \\
e_{i j}=n_{i j}+a_{i j} &
\end{array}
$$

Le capital marque global de la marque j (e.j) s'obtient en calculant la moyenne des valeurs eij, que pondèrent les quantités achetées (qi). Ainsi la contribution de chaque individu au capital de la marque est proportionnelle à sa consommation du produit ${ }^{2}$, soit

$$
e_{. j}=\frac{1}{Q} \sum_{i=1}^{N} e_{i j} \cdot q_{i}
$$

$\mathrm{q}_{\mathrm{j}}$ : $\quad$ quantité du produit consommée par l'individu i sur une période de référence.

Q : $\quad$ quantité totale du produit consommée par l'échantillon sur la même période.

\section{APPLICATION A LA MESURE DU DIFFERENTIEL DE PART DE MARCHE EXPLIQUE PAR LA MARQUE}

La mesure du capital marque e.j s'appuyant sur un calcul de différence d'utilités, l'application opérationnelle qui en dérive est celle du calcul de la part de marché théorique qu'explique la marque. La démarche étant ici similaire à celle de Park et Srinivasan (1994), nous nous contentons d'en rappeler brièvement les étapes.

Le capital d'une marque de distributeur est ici utilisé comme référence. Soulignons que la marque de distributeur peut être utilement remplacée par une marque fictive ou par toute autre marque utilisée comme "benchmark» et qui posséderait les mêmes caractéristiques que celles prêtées à la marque de distributeur. La marque de distributeur peut en effet sur certains marchés prétendre aux caractéristiques d'une marque à part entière et donc revendiquer un capital propre. La méthode statistique que nous utilisons nécessite seulement l'utilisation d'une marque de référence incontestable dont le capital marque soit le plus faible possible.

Dans l'expérimentation dont nous présentons les résultats, aucune mesure de la quantité achetée ou de la fréquence d'achat n'a été effectuée. C'est pourquoi nous considérons par la suite que tous les individus ont un volume de consommation identique. Nous calculons donc une moyenne non pondérée sur la totalité de l'échantillon pour chaque marque j. De façon générale, nous préconisons de pondérer les valeur $\mathrm{e}_{\mathrm{ij}}$ par les quantités $\mathrm{q}_{\mathrm{i}}$ uniquement pour le calcul du capital marque global : lorsqu'il s'agit de comparer des mesures inter-individus, il est préférable de partir des mesures non pondérées. 
Nous posons dès lors la définition suivante :

$\mathbf{D}_{4}-$ Le différentiel de part de marché qu'explique la marque est la différence entre la part de marché de la marque et celle qu'obtiendrait le même produit commercialisé sous une marque de distributeur.

qui conduit à l'équation (10) :

$$
\Delta M K S_{j}=M K S(a)_{j}-M K S(d)_{j}
$$

$\triangle \mathrm{MKS}_{\mathrm{j}}$

La première étape réside dans le calcul de la quantité $\operatorname{MKS}(\mathrm{a})_{\mathrm{j}}$. Soit $\operatorname{Pr}_{\mathrm{ij}}$ la probabilité pour un sujet $\mathrm{i}$ de choisir la marque $\mathrm{j}$. La moyenne des $\operatorname{Pr}_{\mathrm{ij}}$ que pondère la quantité achetée $\mathrm{q}_{\mathrm{i}}$ exprime la part de marché de $\mathrm{j}$. $\operatorname{Pr}_{\mathrm{ij}}$ est calculée à l'aide d'un modèle de régression Logit qui estime la probabilité d'achat d'un produit en fonction de son utilité globale ${ }^{3}$. L'expression générale de $\operatorname{Pr}_{\mathrm{ij}}$ devient alors :

$$
\operatorname{Pr}_{i j}=\frac{\exp \left\{\beta \cdot u(s)_{i j}\right\}}{\sum_{j=1}^{n} \exp \left\{\beta \cdot u(s)_{i j}\right\}}=\frac{\exp \left\{\beta \cdot\left[u(o)_{i j}+e_{i j}\right]\right\}}{\sum_{j=1}^{n} \exp \left\{\beta \cdot\left[u(o)_{i j}+e_{i j}\right]\right\}}
$$

dans laquelle $\beta$ est un paramètre dont la valeur maximise la fonction du maximum de vraisemblance L suivante (Silk et Urban, 1978) :

$$
\mathrm{L}=\prod_{\mathrm{i}=1}^{\mathrm{N}} \prod_{\mathrm{j}=1}^{\mathrm{n}}\left(\operatorname{Pr}_{\mathrm{ij}}\right)^{\delta_{\mathrm{ij}}}
$$

Sij : valeur égale à 1 si l'individu i a effectivement acheté la marque j pendant la période de référence sinon valeur égale à 0 .

En remplaçant dans l'équation (11) et pour chaque individu i, le capital de la marque $\mathrm{j}$ (soit $\mathrm{e}_{\mathrm{ij}}$ ) par $\mathrm{e}_{\mathrm{id}}$, la le capital de la marque de distributeur choisie comme «benchmark», nous calculons $\operatorname{Pr}(\mathrm{d})_{\mathrm{ij}}$, autrement dit la probabilité pour l'individu i de choisir le produit $\mathrm{j}$, si celui-ci avait une valeur de capital marque égale à celle de la marque de distributeur d. La part de marché $\mathrm{MKS}(\mathrm{d}) \mathrm{j}$ est alors égale à la moyenne des $\operatorname{Pr}(\mathrm{d})_{\mathrm{ij}}$ pondérée par les quantités achetées $q_{i}$. Nous disposons dès lors des deux termes de l'équation (10), afin de calculer le différentiel de part de marché attribuable à la marque j.

Avant d'aborder la comparaison entre la méthode d'expérimentation que nous préconisons et celle mise en œuvre par Park et Srinivasan (1994), nous récapitulons nos variables de mesure (cf. tableau 1).

Dans l'hypothèse où la personne interrogée achète une marque différente de l'ensemble des marques évaluées, la probabilité d'achat de l'ensemble des « autres marques » est calculée par la fonction suivante dans laquelle un paramètre $\alpha$ à estimer remplace

$$
\text { l'expression } \exp \left\{\beta \cdot \mathrm{u}(\mathrm{s})_{\mathrm{ij}}\right\}: \operatorname{Pr}_{\mathrm{ij}}=\frac{\alpha}{\sum_{\mathrm{j}=1}^{\mathrm{j}=\mathrm{n}-1} \exp \{\beta \cdot \mathrm{u}(\mathrm{s}) \mathrm{ij}\}+\alpha} \text {. Le paramètre } \alpha \text { est estimé par un algorithme de détermination }
$$

itérative du couple $(\alpha ; \beta)$ maximisant la fonction $\mathrm{L}$. 
TABLEAU 1

DEFINITION DES VARIABLES DE MESURE UTILISEES

\begin{tabular}{|c|c|c|}
\hline Libellé & Description & Expression \\
\hline $\mathrm{u}(\mathrm{s})_{\mathrm{ij}}$ & Utilité pour l'individu i du produit j avec la marque & - \\
\hline $\mathrm{u}(\mathrm{o})_{\mathrm{ij}}$ & Utilité pour i du produit j sans la marque & - \\
\hline $\mathrm{v}(\mathrm{s})_{\mathrm{ij}}$ & Utilité pour i des attributs du produit j avec la marque & - \\
\hline $\mathrm{v}(\mathrm{o})_{\mathrm{ij}}$ & Utilité pour i des attributs du produit j sans la marque & - \\
\hline $\mathrm{e}_{\mathrm{ij}}$ & Capital marque individuel selon l'auteur & $\mathrm{e}_{\mathrm{ij}}=\mathrm{u}(\mathrm{s})_{\mathrm{ij}}-\mathrm{u}(\mathrm{o})_{\mathrm{ij}}$ \\
\hline $\mathrm{e}_{\mathrm{ij}}$ & Capital marque individuel selon Park et Srinivasan & $\mathrm{e}_{\mathrm{ij}}^{\prime}=\mathrm{u}(\mathrm{s})_{\mathrm{ij}}-\mathrm{v}(\mathrm{o})_{\mathrm{ij}}$ \\
\hline$\xi(0)_{i j}^{[1]}$ & Terme d'erreur lorsque la marque est occultée & $\xi(\mathrm{o})_{\mathrm{ij}}=\left[\mathrm{e}_{\mathrm{ij}}-\mathrm{e}_{\mathrm{ij}}\right]=\mathrm{u}(\mathrm{o})_{\mathrm{ij}}-\mathrm{v}(\mathrm{o})_{\mathrm{ij}}$ \\
\hline$\xi(\mathrm{s})_{\mathrm{ij}}$ & Terme d'erreur lorsque la marque est présente & $\xi(\mathrm{s})_{\mathrm{ij}}=\xi(\mathrm{o})_{\mathrm{ij}}$ \\
\hline $\mathrm{a}_{\mathrm{ij}}$ & Capital marque individuel fondé sur les attributs du produit & $\mathrm{a}_{\mathrm{ij}}=\mathrm{v}(\mathrm{s})_{\mathrm{ij}}-\mathrm{v}(\mathrm{o})_{\mathrm{ij}}$ \\
\hline \multirow[t]{2}{*}{$\mathrm{n}_{\mathrm{ij}}$} & Capital marque individuel non fondé sur les attributs du produit & $\mathrm{n}_{\mathrm{ij}}=\left[\mathrm{u}(\mathrm{s})_{\mathrm{ij}}-\mathrm{v}(\mathrm{s})_{\mathrm{ij}}\right]-\xi(\mathrm{s})_{\mathrm{ij}}$ \\
\hline & Equation fondamentale : & $\mathbf{e}_{\mathrm{ij}}=\mathbf{a}_{\mathrm{ij}}+\mathbf{n}_{\mathrm{ij}}$ \\
\hline $\operatorname{MKS}(\mathrm{a})_{\mathrm{j}}$ & Part de marché de la marque d'annonceur j & \\
\hline $\operatorname{MKS}(d)_{j}$ & Part de marché du produit j vendu sous marque de distributeur & \\
\hline$\Delta \mathrm{MKS}_{\mathrm{j}}$ & Différentiel de part de marché expliqué par la marque j & $\Delta \mathrm{MKS}_{\mathrm{j}}=\operatorname{MKS}(\mathrm{a})_{\mathrm{j}}-\mathrm{MKS}(\mathrm{d})_{\mathrm{j}}$ \\
\hline
\end{tabular}

${ }^{[1]}$ Rappel : ce terme d'erreur ainsi que celui qui suit reflète la différence qui existe entre la préférence du consommateur mesurée d'une façon directe et celle calculée à l'aide d'un modèle multi-attributs.

\section{C)- MÉTHODOLOGIE D'EXPÉRIMENTATION}

Afin de préserver l'intérêt d'une mesure individuelle du capital marque, nous faisons le choix d'un plan d'expérience à mesures répétées : chaque sujet évalue deux fois le même produit dont on manipule la marque en absence-présence. A la différence de Park et Srinivasan (1994), qui interrogent deux populations - l'une formée de consommateurs, l'autre d'experts - nous n'interrogeons qu'un seul échantillon. Rappelons que les deux auteurs estiment que les dentistes, en qualité d'experts, ne sont pas influencés par la marque et qu'ils évaluent donc objectivement les attributs du dentifrice et du rince-bouche. On peut toutefois critiquer cette assertion (Brucks, 1985 ; Alba et Hutchinson, 1987) et soutenir que le professionnel subit lui aussi l'influence de la marque : n'est-il pas la cible d'actions commerciales et marketing spécifiques de la part du fabricant ? Bref, quelle que soit la population interrogée, il paraît difficile d'isoler sans recourir à une manipulation en absence-présence (Olson, 1977) l'influence spécifique de la marque. Ajoutons d'autre part que le choix méthodologique des deux auteurs les contraint à calculer une différence d'utilités à un niveau agrégé : les deux mesures portent en effet sur des populations différentes. En résumé, la méthode expérimentale que nous recommandons, outre qu'elle garantit un meilleur contrôle des facteurs de distorsion éventuelle, préserve l'intérêt d'une mesure individuelle du capital marque : le plan d'expérience à mesures répétées présente a priori une meilleure validité interne et externe que la confrontation de deux évaluations qualifiées respectivement « de subjective » (consommateurs) et « d'objective» (experts), sous réserve toutefois de contrôler les facteurs de distorsion qu'introduisent inévitablement la fatigue, 
l'apprentissage ou bien encore la recherche d'une cohérence excessive des répondants (cf. page 12). Dans le cadre de l'expérimentation, les répondants ont été invités à une heure à leur convenance, prévenus de la durée de l'interrogation et enfin, sollicités pour donner leur opinion sur différents produits présentés aléatoirement avec ou sans mention de la marque et susceptibles d'être tous différents. Les enquêteurs eux-mêmes n'étaient pas au courant de la logique poursuivie.

\section{4)- VALIDATION EXPERIMENTALE ET PRESENTATION DES RESULTATS}

Nous pouvons raisonnablement estimer que la justification théorique et méthodologique développée précédemment nous assure de la bonne validité interne de l'instrument de mesure : sa validité de trait et de critère ne seront donc pas reprise dans le cadre de cet article. En revanche, il convient de comparer la validité externe de notre méthode avec celle de Park et Srinivasan (1994) : cette démarche fait l'objet de notre expérimentation. Nous mettrons en évidence l'incidence du terme d'erreur en comparant les résultats de notre mesure et de celle de Park et Srinivasan (1994) avant d'examiner la validité prédictive des deux mesures. Pour cela, nous isolons le terme d'erreur qui résulte de la différence de calcul entre les deux approches. Puis, nous montrons que le capital marque, estimé selon notre méthode, est plus fortement corrélé avec des construits proches d'un point de vue théorique : la part de marché, la distribution numérique et la distribution valeur. Enfin, nous calculons sur un exemple la part de marché théorique qu'explique la marque, afin d'illustrer l'intérêt opérationnel de notre démarche.

\section{A)- L'EXPÉRIMENTATION}

Un échantillon de 180 personnes est convié sur Paris à un test de dégustation et d'évaluation de produits. Les quotas de recrutement portent sur le genre, l'âge et la catégorie socioprofessionnelle. Aucun quota n'a été imposé sur la fréquence et le volume de consommation: on peut estimer que la qualité de représentativité de l'échantillon n'est pas fortement remise en cause dans la mesure où les produits retenus sont d'un usage courant. L'expérimentation porte sur 8 catégories de produits : 4 biens durables (le lave-linge, le micro-ordinateur, le téléviseur, la carte de crédit) et 4 biens de consommation (le café soluble, le champagne, le chocolat noir et la crème glacée). 4 marques par produit directement comparables en termes de performances, de fonctionnalités et de prix ont été choisies. Leur notoriété, leur part de voix publicitaire, leur distribution numérique et leur distribution valeur nous assurent de leur large diffusion sur le marché. De plus, une marque de distributeur dans le cas de la crème glacée est incluse.

Pour chaque catégorie de produit, nous identifions au préalable une liste des attributs déterminants. Nous adoptons la méthode du questionnaire dual reconnue pour sa simplicité et sa fiabilité (Alpert, 1971). Parce que sa mise en oeuvre conduit à retenir un nombre trop élevé de critères de choix (Alpert, 1980), nous apurons la liste initiale en nous assurant que les attributs sont indépendants entre eux (Beckwith et Lehman, 1975). La liste finale contient 3 à 5 critères de choix par catégorie de produit : ainsi constituée la liste est conforme au nombre de stimuli qu'un consommateur peut raisonnablement assimiler (Feldman et Lynch, 1988). 


\section{STRUCTURE DU QUESTIONNAIRE ET MATERIEL EXPERIMENTAL}

Le questionnaire est administré par ordinateur. Dans la mesure où la structure du questionnaire est proche de celle utilisée par Park et Srinivasan (1994), nous nous contentons de résumer brièvement son contenu. La première partie détermine la fonction d'utilité de chaque personne interrogée (Green et Srinivasan, 1990). Nous mesurons dans un premier temps la préférence individuelle entre les différentes modalités de chaque attribut, puis dans un deuxième temps l'importance relative qu'accorde chaque individu aux attributs :

- Préférence individuelle entre les modalités : pour les attributs dont les modalités sont discrètes, le répondant classe chaque modalité selon sa préférence : la plus appréciée reçoit la note 10, la moins appréciée la note 1 , les modalités restantes étant évaluées entre ces deux bornes. La préférence pour les autres attributs, considérés comme des variables continues, s'exprime à l'aide d'un modèle vectoriel linéaire (Green et Srinivasan, 1978). A l'exception du prix, les modalités extrêmes de ces variables continues sont dénommées respectivement «très satisfaisant» et « acceptable ». Pour la variable prix, le prix le moins élevé et le prix le plus élevé de la catégorie de produit définissent les bornes inférieures et supérieures.

- Importance relative de chaque attribut: le répondant choisit en premier lieu un attribut critique. Sa détermination procède d'une série de comparaisons par paires de différentes propositions (Srinivasan, 1988) : chaque proposition substitue pour un attribut la modalité la moins appréciée à celle qui est la plus appréciée issue de la phase précédente. L'attribut critique, qui ressort de la proposition finalement retenue, se voit attribuer une note de 10 points. Les autres attributs sont alors évalués sur une échelle d'importance de 1 à 10 en utilisant l'attribut critique comme référence.

La deuxième et la troisième parties correspondent aux phases d'évaluation des produits, la marque étant successivement occultée puis mentionnée. Elles se décomposent en deux étapes : une évaluation des attributs à l'aide d'une note de 1 à 10 - à l'exception des attributs à modalités discrètes et du prix compte tenu de leur caractère objectif - et une évaluation globale qui s'inspire de la méthode connue sous le nom de «dollars métrique ». A l'instar de Park et Srinivasan (1994), nous choisissons une procédure simplifiée, en raison du nombre trop élevé de comparaisons par paires que suppose le recours à la méthode complète (48) préconisée par Pessemier et al (1971) : le répondant choisit le produit qu'il apprécie le plus et celui qu'il apprécie le moins, auxquels sont attribués les notes 10 et 1 . Puis, il évalue les deux autres produits à l'aide de la même échelle. Enfin, le sujet est interrogée sur l'écart de prix qu'il est prêt à payer pour acheter le produit qu'il apprécie le plus à la place de l'avant dernier produit qu'il apprécie le moins. Une transformation linéaire permet d'obtenir l'écart de prix entre les quatre marques pour chaque produit, conformément à la formule (13) ci-dessous : 


$$
U_{i j}=\frac{\Delta P_{i}}{10-m_{i}} x R_{i j}
$$

$\mathrm{U}_{\mathrm{ij}} \quad$ : $\quad$ utilité exprimée en unité monétaire de la marque j pour l'individu i.

$\Delta \mathrm{P}_{\mathrm{i}} \quad: \quad$ écart de prix communiqué par l'individu $\mathrm{i}$.

$\mathrm{R}_{\mathrm{ij}} \quad$ : $\quad$ note d'évaluation globale de la marque $\mathrm{j}$ attribuée par l'individu $\mathrm{i}$.

$\mathrm{m}_{\mathrm{i}} \quad$ : note d'évaluation globale de la seconde marque la moins appréciée attribuée par l'individu i.

Les raisons du choix de la marque appréciée le plus font l'objet d'une question ouverte. Enfin, une dernière partie recueille les dernières marques achetées ou possédées, afin de calibrer notre modèle de choix probabiliste.

Le matériel expérimental varie selon les produits. Pour les biens alimentaires, les personnes interrogées goûtent les produits et consultent leurs caractéristiques organoleptiques. Lors de l'évaluation avec la mention de la marque, les visuels présentent l'emballage du produit sur lequel figure la marque. Les biens durables sont évalués à partir des photographies des produits et de la mention de leurs principales caractéristiques. La marque est naturellement occultée lors de la phase d'évaluation en « aveugle».

\section{B)- VALIDATION EXPÉRIMENTALE : LES RÉSULTATS}

La méthode de calcul du capital marque $\mathrm{e}_{\mathrm{ij}}$ telle que nous l'avons définie isole un terme d'erreur $\xi(\mathrm{o})_{\mathrm{ij}}$. L'estimation de son ordre de grandeur tente de répondre à deux questions fondamentales : le modèle multiattribut additif retenu permet-il de prédire avec certitude la marque que choisit le consommateur ? Sa validité prédictive est-elle vérifiée quel que soit le produit? Si nous démontrons que la valeur de $\xi(0)_{\mathrm{ij}}$ n'est pas négligeable au regard de celle de $\mathrm{e}_{\mathrm{ij}}$, nous justifions le bien-fondé de la correction que nous apportons au modèle de Park et Srinivasan (1994).

\section{L'INCIDENCE DU TERME D'ERREUR $\xi(O)_{\text {IJ }}$}

L'annexe 1 récapitule les valeurs de $\mathrm{e}_{\mathrm{ij}}$ calculées d'après les équations (2) et (3). La différence entre les résultats issus de l'application des deux méthodes de calcul est égale au terme d'erreur $\xi(\mathrm{o})_{\mathrm{ij}}$. Le ratio $\Omega_{\mathrm{ij}}$ ci-dessous exprime ce terme d'erreur en proportion de la valeur absolue de $\mathrm{e}_{\mathrm{ij}}: \Omega_{\mathrm{ij}}$ exprime donc la part d'erreur contenu dans la mesure $\mathrm{du}$ capital marque. Rappelons que $\xi(0)_{i j}$ et $e_{i j}$ sont exprimés en unité monétaire comme l'ensemble des utilités (cf. équation 13) : leurs valeurs sont donc dépendantes du prix moyen de la catégorie de produit. Exprimer $\xi(\mathrm{o})_{\mathrm{ij}}$ en proportion du capital marque $\left(\mathrm{e}_{\mathrm{ij}}\right)$ rend ainsi possible une comparaison entre les différentes catégories de produits :

$$
\Omega_{\mathrm{ij}}=\left|\frac{\xi(\mathrm{o})_{\mathrm{ij}}}{\mathrm{e}_{\mathrm{ij}}}\right|
$$

L'examen des valeurs de $\xi(0)_{i j}$ montre que la correction proposée n'est pas négligeable. Son signe est soit positif, soit négatif : selon les marques, l'utilité calculée à l'aide du modèle multiattribut majore ou minore la préférence exprimée directement par le sujet. La valeur élevée des écarts types révèle que le terme d'erreur varie fortement entre les sujets : la contribution de sa composante aléatoire (propre à chaque individu) l'emporte sur celle de sa composante systématique (partagée entre tous les sujets). La forte 
hétérogénéité du terme d'erreur d'une marque à l'autre plaide en faveur de l'utilisation de notre méthode de calcul dont la fiabilité s'avère en première analyse meilleure que celle de Park et Srinivasan (1994). En effet, nous pourrions estimer que l'erreur de mesure a un impact minime si sa valeur était identique pour toutes les marques au sein d'une catégorie de produit, puisque la variable $e_{\mathrm{ij}}$, dont la somme sur $\mathrm{j}$ est égale à 0 , n'est déterminée qu'à une constante près. Cette condition ne s'avère pas remplie : la quantité $\xi(\mathrm{o})_{\mathrm{ij}}$ varie par exemple entre -259,06 (Vedette) et 367,95 (Whirpool) dans le cas du lave-linge. L'analyse est encore plus éloquente si nous considérons la moyenne sur les individus i des quantités $\Omega_{\mathrm{ij}}$. Dans ce cas, le maximum de $\Omega_{\mathrm{j}}$ atteint 3,49 (Thomson), la valeur minimum étant de 0,89 (Carte Noire) : le terme d'erreur $\xi(0)$.j est pour certaines marques plus élevé que leur valeur de capital marque.

En conclusion, ces premiers résultats plaident en faveur de la correction que nous apportons. La validité interne de notre mesure s'avère meilleure que celle de Park et Srinivasan (1994), puisqu'elle neutralise une proportion de variance non négligeable d'ordre instrumental. Il convient maintenant de discuter de la validité externe de notre instrument de mesure et plus particulièrement de sa validité prédictive. Pour cela, deux démarches sont menées : la première étudie la corrélation entre la mesure de capital marque et celle d'autres indicateurs de la force de la marque; la seconde compare la part de marché prédite par le modèle Logit construit à partir de notre mesure et celle donnée par un panel.

\section{LA CORRELATION ENTRE LE CAPITAL MARQUE ET D'AUTRES INDICATEURS DE LA FORCE DE LA MARQUE}

S'assurer de la validité prédictive de notre méthode révisée revient en premier lieu à démontrer que notre mesure du capital marque est logiquement corrélée à d'autres indicateurs de la force de la marque, proches d'un point de vue théorique (Nunnally, 1978). Pour cela, nous retenons deux familles d'indicateurs, la part de marché (Aaker, 1991) et le taux de distribution (Keller, 1993 ; Riezebos, 1994), mesurées chacune par deux variables : la part de marché en volume (PdM vol.) et la part de marché en valeur (PdM val.), l'indice de distribution numérique (DN) et l'indice de distribution valeur (DV). En effet, les marques qui jouissent d'un capital marque élevé occupent habituellement une position privilégiée : leurs parts de marché et leurs indices de distribution sont en général supérieurs à la moyenne (Aaker, 1992 ; Kamakura et Russell, 1993). Afin de comparer les valeurs de capital marque entre les produits, nous exprimons chaque variable $\mathrm{e}_{\mathrm{ij}}$ en pourcentage du prix de vente moyen de sa catégorie, puis nous centrons l'ensemble des indicateurs retenus (cf. annexe 2).

Le tableau 2 reprend les coefficients de corrélation calculés sur l'ensemble des produits, puis pour les biens de consommation et les biens durables séparément. Les résultats révèlent que, sur la totalité des produits, quelle que soit la méthode de valorisation retenue, les corrélations entre la valeur de capital marque et les autres indicateurs de la force de la marque sont non significatives, à l'exception de la relation positive entre notre mesure du capital marque et la part de marché valeur $(\rho=0,41 ; p<$ $0,05)$. Il est vrai que les parts de marché (aussi bien en volume qu'en valeur) de certaines catégories de produits - en particulier le café soluble et le champagne - sont faibles, soit parce que le marché est très " atomisé » (le café soluble), soit parce que les fabricants optent pour une politique de distribution sélective (le champagne). Cette 
particularité est propre à altérer l'intensité de la corrélation que nous cherchons à mesurer. Enfin, il est difficile de généraliser les résultats observés car ceux-ci ne portent que sur un faible nombre de marques (32). On peut aussi penser qu'il est difficile de généraliser la relation positive entre le capital marque et la part de marché à tous les produits : certaines marques « haut de gamme » - a priori absente dans notre expérimentation - n'ont-elles pas la particularité de disposer d'un capital marque élevé et d'une part de marché de «niche»?

TABLEAU 2

COEFFICIENTS DE CORRELATION ENTRE LES VALEURS DE CAPITAL MARQUE, LES PARTS DE MARCHE ET LES INDICES DE DISTRIBUTION NUMERIQUE ET DE DISTRIBUTION VALEUR ${ }^{(a)}$

\begin{tabular}{|c|c|c|c|c|c|c|c|c|}
\hline \multirow{2}{*}{$\begin{array}{l}\mathrm{N}=32 \text { observations } \\
4 \text { marques / produit } \\
4 \text { produits / catégorie } \\
2 \text { catégories }\end{array}$} & \multicolumn{4}{|c|}{ Méthode de Park et Srinivasan (1994) } & \multicolumn{4}{|c|}{ Méthode révisée } \\
\hline & $\begin{array}{c}\text { PdM } \\
\text { vol. }\end{array}$ & $\begin{array}{c}\text { PdM } \\
\text { val. }\end{array}$ & $\mathrm{DN}$ & DV & $\begin{array}{c}\text { PdM } \\
\text { vol. }\end{array}$ & $\begin{array}{c}\text { PdM } \\
\text { val. }\end{array}$ & $\mathrm{DN}$ & DV \\
\hline \multicolumn{9}{|l|}{ Catégories } \\
\hline Biens de consommation & $0,52 \quad(n . s)$ & $0,34 \quad$ (n.s) & $-0,02 \quad(n . s)$ & $-0,02$ (n.s) & 0,19 (n.s) & $0,06 \quad$ (n.s) & $0,04 \quad$ (n.s) & 0,04 (n.s) \\
\hline Biens durables & $0,58 \quad(\mathrm{n} . \mathrm{s})$ & 0,65 & $0,43 \quad(\mathrm{n} . \mathrm{s})$ & 0,33 (n.s) & 0,67 & $0,72(* *)$ & $0,62 \quad(*)$ & $0,78(* *)$ \\
\hline Total des produits & $0,37 \quad$ (n.s) & 0,31 & $0,06 \quad(n . s)$ & $0,08 \quad$ (n.s) & 0,34 (n.s) & $0,41 \quad(*)$ & 0,13 & $0,28 \quad$ (n.s) \\
\hline $\begin{array}{ll}\text { Légende: } & \text { (a) } \\
& (*) \\
& (* *)\end{array}$ & $\begin{array}{l}\text { Les valeurs s } \\
p<0,05 \text {. } \\
p<0,01\end{array}$ & ignificatives & sont souligné & es en gras, l' & sque préci & at le seuil c & significativ & \\
\hline
\end{tabular}

Toutefois, s'agissant des biens durables, notre mesure du capital marque est positivement corrélée avec chacun des autres indicateurs retenus, alors que ces mêmes corrélations ne sont pas significatives dans le cas de Park et Srinivasan. L'appartenance aux biens durables semble exercer un effet significatif sur la relation entre le capital marque et les autres mesures de la force de la marque, ce que confirme partiellement une analyse de variance. La catégorie de produit, considérée comme une variable indépendante, exerce un effet modérateur sur la corrélation entre le capital marque et la part de marché (en valeur uniquement), et ce quelle que soit la méthode de valorisation utilisée $(F=9,96 ; p=0,013)$. En résumé, sans prétendre généraliser notre conclusion à tous les produits, nous pouvons affirmer que notre mesure du capital marque est plus fortement corrélée aux indicateurs habituellement retenus pour mesurer la force de la marque que ne l'est celle de Park et Srinivasan (1994) : sa validité prédictive semble être, de ce fait, meilleure.

\section{LA COMPARAISON ENTRE LA PART DE MARCHE CALCULEE, DECLAREE ET MESUREE PAR UN PANEL}

Afin de montrer que notre construit de capital marque reflète correctement la valeur actuelle de la marque sur le marché, nous comparons pour la crème glacée trois types de données (cf. tableau 3) : la part de marché issue du panel, la part de marché calculée d'après la dernière marque achetée et enfin la part de marché obtenue par l'application du modèle Logit (cf. équation 11) construit à partir de la mesure individuelle du capital marque. Nous y ajoutons le calcul du différentiel de part de marché qu'explique la marque (cf. équation 10).

\section{TABLEAU 3}


COMPARAISON DES VALEURS DE PARTS DE MARCHE REELLES, DECLAREES ET CALCULEES.

\begin{tabular}{|c|c|c|c|c|c|c|}
\hline $\begin{array}{l}\text { Marques de crèmes } \\
\text { glacées }\end{array}$ & $\begin{array}{c}\mathrm{PdM} \\
\text { Iri-Secodip }\end{array}$ & $\begin{array}{c}\text { PdM } \\
\text { déclarée }\end{array}$ & $\begin{array}{c}\text { PdM } \\
\text { calculée } \\
\operatorname{MKS}(a)_{j}\end{array}$ & $\begin{array}{l}\text { PdM ajustée } \\
\text { MKS }(d)_{j}\end{array}$ & \multicolumn{2}{|c|}{$\begin{array}{c}\text { Différentiel de PdM } \\
\text { expliqué par la marque } \\
\Delta \mathrm{MKS}_{\mathrm{j}}\end{array}$} \\
\hline$(\mathrm{N}=198$ personnes $)$ & (1) & (2) & (3) & (4) & (4)-(3) & En \% de (3) \\
\hline Instants choisis de Miko & 13,20 & 11,96 & 15,72 & 14,04 & 1,68 & 10,68 \\
\hline Exquise de Gervais & 14,30 & 14,31 & 14,30 & 10,89 & 3,41 & 23,84 \\
\hline Côte d'Or de Motta & 20,10 & 25,67 & 18,17 & 12,87 & 5,30 & 29,17 \\
\hline Distributeur Carrefour & 22,50 & 16,07 & 20,07 & - & - & \\
\hline Autres marques / aucune & 29,90 & 31,99 & 31,72 & - & - & \\
\hline Total & 100,00 & 100,00 & 100,00 & & & \\
\hline $\begin{array}{l}\text { (1) } \\
\text { (2) } \\
\text { (3) } \\
\text { (4) }\end{array}$ & $\begin{array}{l}\text { Source Iri-Secodip d } \\
\text { Sur la base de la den } \\
\text { Calculée à l'aide du } \\
\text { Part de marché théor }\end{array}$ & $\begin{array}{l}\text { és panels } \\
\text { marque ac } \\
\text { èle de régre } \\
\text { du produit }\end{array}$ & $\begin{array}{l}\text { teurs } 1997 . \\
\text { Logit (équat } \\
\text { nnonceur cr }\end{array}$ & $\begin{array}{l}1 \text { et } 12) \text {. } \\
\text { du capital ma }\end{array}$ & u dist & \\
\hline
\end{tabular}

Nous remarquons que la part de marché calculée $\operatorname{MKS}(\mathrm{a})_{\mathrm{j}}$ est plus fortement corrélée à la part de marché panel $\left(\mathrm{r}^{2}=0,95, \mathrm{p}=0,01\right)$ qu'à la part de marché calculée d'après la dernière marque achetée $\left(\mathrm{r}^{2}=0,85, \mathrm{p}=0,07\right)$. Seule « anomalie », la marque Carrefour dont la part de marché déclarée $(16,07)$ est inférieure à la part de marché panel $(22,50)$, elle-même proche de la part de marché calculée $(20,07)$. Ceci s'explique sans doute par la réticence des sujets à exprimer ouvertement leur préférence pour une marque de distributeur. Notons que la part de marché des «autres marques » est correctement évaluée par le modèle (31,72 contre 29,90 de part de marché réelle), ce qui reflète une bonne estimation de la valeur du paramètre $\alpha$ inclus dans le modèle Logit. Soulignons enfin que la part de marché de chaque marque est d'autant plus élevée que le différentiel de part de marché expliqué par la marque est élevé : il existe sur le marché de la crème glacée une relation significative entre la valeur du capital marque et la part de marché du produit $\left(r^{2}=0,82\right)$. En conclusion, le modèle de prévision, calibré sur notre méthode révisée, paraît offrir une bonne validité externe. Sa capacité de prévision de la part de marché est, sur cet exemple limité à la seule crème glacée, meilleure qu'un calcul fondé sur le relevé de la dernière marque achetée.

Une autre façon de vérifier la validité prédictive de notre mesure est de vérifier que le modèle Logit qui en découle prédit correctement la dernière marque achetée, considérée comme la variable dépendante. Nous faisons l'hypothèse que la marque prédite est celle qui obtient la probabilité de choix $\operatorname{Pr}_{\mathrm{ij}}$ la plus élevée et nous la comparons à la dernière marque réellement achetée. Le modèle Logit prédit correctement la marque achetée pour 125 personnes sur 198 soit $63,1 \%$, un pourcentage significativement plus élevé $(\mathrm{z}=$ $8,01, p=0,000)$ que celui obtenu par un tirage purement aléatoire $(23,2 \%)^{4}$. Ce score est comparable à celui que calcule Park (1992) pour le dentifrice $(69 \%)$ mais il est de 10 points supérieur à celui que l'auteur rapporte pour le rince-bouche $(53 \%)$. Soulignons enfin que la valeur de cet indice d'ajustement est en réalité probablement minorée par la

Soit $\mathrm{m}_{\mathrm{j}}$ la part de marché de la marque $\mathrm{j}(\mathrm{j}=1,2,3,4,5)$ calculée sur la base de la dernière marque achetée (l'indice 5 désignant l'ensemble des autres marques). La probabilité d'un classement correct à l'issue d'un tirage au sort purement aléatoire est égale à $\sum_{\mathrm{j}=1}^{\mathrm{j}=5}(\mathrm{mj})^{2}=(0,11)^{2}+(0,15)^{2}+(0,25)^{2}+(0,16)^{2}+(0,33)^{2}=0,23$. 
sous-déclaration des choix qui se portent sur la marque Carrefour. La qualité d'ajustement de notre modèle Logit est donc satisfaisante, ce que confirme le calcul plus formel d'indices d'entropie (Hauser, 1978). Soit Z l'incertitude totale ou entropie a priori du système $(Z=0,66)$, EI l'information attendue ou réduction de l'entropie par le modèle $(\mathrm{EI}=0,57)^{5}$, le rapport $\mathrm{G}$ de $\mathrm{EI}$ par $\mathrm{Z}$ reflète la part d'incertitude expliquée par le modèle (ou indice d'utilité du modèle) : sur cet exemple, G est égal à 0,86 , ce qui signifie que le modèle explique $86 \%$ de l'incertitude a priori du choix.

Notre mesure du capital marque semble montrer une bonne validité prédictive. Cette conclusion se trouve renforcée par la comparaison entre deux mesures : la proportion de l'échantillon qui cite spontanément la marque comme raison principale de leur choix et les valeurs absolues du capital marque exprimées en pourcentage du prix de vente pour chaque produit (cf. tableau 4). Une constatation, surprenante au premier abord, s'impose : les sujets qui invoquent la marque pour justifier leur préférence sont moins nombreux dans le cas des produits de grande consommation, dont la valeur relative de capital marque est pourtant la plus élevée ! En réalité, pour ces produits, il est vraisemblable que les personnes interrogées sont plus réticentes à avouer que leur choix repose sur une préférence subjective pour la marque : parce qu'ils ont la possibilité de goûter le produit, leur sensibilité gustative intervient certainement pour motiver leur préférence et les inciter à se référer, de façon quasi-exclusive, aux qualités organoleptiques du produit (Smith et Swinyard, 1982).

\section{TABLEAU 4}

COMPARAISON DE LA VALEUR RELATIVE DU CAPITAL MARQUE ET DU POURCENTAGE DE L'ECHANTILLON AYANT CITE LA MARQUE COMME RAISON DE LEUR PREFERENCE.

\begin{tabular}{|c|c|c|c|c|}
\hline \multirow[t]{3}{*}{ Catégories de produits } & \multicolumn{2}{|c|}{$\underline{\text { Mesure indirecte }}$} & \multicolumn{2}{|c|}{$\underline{\text { Mesure directe }}$} \\
\hline & \multicolumn{2}{|c|}{$\begin{array}{l}\text { Valeur absolue du capital marque en } \% \\
\text { du prix de vente }\end{array}$} & \multicolumn{2}{|c|}{$\begin{array}{l}\text { Nombre de sujets citant la marque } \\
\text { comme raison de préférence }\end{array}$} \\
\hline & $\%$ & $\sigma$ & Effectif & $\%$ \\
\hline Biens de consommation : & $\underline{\underline{19,04}}$ & - & - & $\underline{\underline{3,5}}$ \\
\hline Chocolat noir en tablette & 25,55 & 13,10 & 8 & 4,0 \\
\hline Champagne brut & 18,38 & 9,71 & 6 & 3,0 \\
\hline Café soluble & 11,33 & 6,59 & 9 & 4,5 \\
\hline Crème glacée & 20,93 & 10,98 & 5 & 2,5 \\
\hline Biens durables: & $\underline{\underline{13,84}}$ & - & - & $\underline{\underline{15,5}}$ \\
\hline Carte de crédit bancaire & $\overline{32,57}$ & 25,34 & 27 & $\overline{13,6}$ \\
\hline Micro-ordinateur & 7,22 & 5,37 & 28 & 14,1 \\
\hline Lave-linge & 6,51 & 4,72 & 22 & 11,1 \\
\hline Téléviseur & 9,09 & 5,50 & 46 & 23,2 \\
\hline
\end{tabular}

Légende $: \mathrm{n}=198$ personnes.

A contrario, pour les biens durables, le mode de présentation des produits, un prix d'achat plus élevé et une implication plus forte des sujets sont autant de facteurs qui accroissent la contribution de la marque elle-même, ce que reconnaissent spontanément les sujets. Ces deux raisons nous permettent d'affirmer qu'une mesure indirecte du rôle de la marque peut s'avérer dans certaines conditions plus robuste qu'une mesure qui repose sur l'interprétation des réponses à une question ouverte.

Soit $\mathrm{P}_{\mathrm{j}}$ la probabilité de choix de la marque j calculée sur la base du dernier achat rapporté et $\mathrm{P}_{\mathrm{ij}}$ la probabilité de choix calculée par le modèle pour chaque individu $i$, alors $Z=-\sum_{j=1}^{j=n} P^{\prime}{ }_{j} \times \log \left(P^{\prime}{ }_{j}\right)$ et $E I=\sum_{i=1}^{i=N} \sum_{j=1}^{j=n} P_{i j} \times \log \left(\frac{P_{i j}}{P^{\prime}{ }_{j}}\right)$. 


\section{INTERET OPERATIONNEL POUR LE PRATICIEN : UN EXEMPLE D'APPLICATION}

Après avoir tenté de montrer que notre méthode de mesure du capital de la marque témoigne d'une bonne validité, à la fois interne et externe (tout au moins prédictive), il convient maintenant de nous interroger sur l'intérêt d'une telle mesure pour le praticien. Pour ce faire, nous commentons les résultats d'une segmentation d'après les avantages recherchés (Green et al., 1985). En effet, si une mesure agrégée du capital de la marque peut être considérée comme un indicateur fiable de la valeur d'une marque, seule une mesure individuelle permet au gestionnaire d'un portefeuille de marques d'optimiser l'allocation de ses ressources marketing.

Le tableau 5 présente les résultats de l'analyse typologique hiérarchique selon l'algorithme de Ward effectuée sur la note d'importance accordée à chaque critère de choix dans l'univers de la crème glacée (la marque ne fait pas partie des variables de segmentation; elle est introduite dans un deuxième temps comme variable explicative des groupes). Au préalable, les scores d'importance sur les $\mathrm{p}$ attributs sont centrés pour chaque individu de telle sorte que leur somme est nulle pour tout individu i. Après avoir effectué une typologie en 2,3, 4 et 5 classes, nous retenons une solution optimum en 4 segments distincts (dont 3 ont des tailles comparables), ce que corroborent les résultats de l'analyse discriminante (lamda de Wilk $=0,09 ; \mathrm{p}=0,00$ ). Chaque segment peut être caractérisé par des attentes spécifiques quant au choix d'une crème glacée : le segment I valorise l'absence de colorants ajoutés au produit, tandis que le segment II privilégie la restitution du vrai goût des ingrédients d'origine. Les segments III et IV recherchent tous deux un produit sans colorant, mais si le premier cite également l'onctuosité du produit, le second insiste davantage sur la recherche du vrai goût des ingrédients d'origine. 
TABLEAU 5

\section{SEGMENTATION SELON LES AVANTAGES RECHERCHES : SCORES D'IMPORTANCE PAR CRITERE DE CHOIX ET VALEUR DE CAPITAL MARQUE PAR SEGMENTS DANS L'UNIVERS DE LA CREME GLACEE.}

\begin{tabular}{|c|c|c|c|c|c|c|c|c|}
\hline \multirow{4}{*}{$\begin{array}{l}\mathrm{N}=198 \text { personnes } \\
\text { Rappel : les variables sont centrées } \\
\text { Taille du segment } \mathbf{( \% )} \\
\text { Bénéfices attendus : }\end{array}$} & \multicolumn{8}{|c|}{ Segments de marché ${ }^{(1)}$} \\
\hline & \multicolumn{2}{|c|}{$\mathbf{I}$} & \multicolumn{2}{|c|}{ II } & \multicolumn{2}{|c|}{ III } & \multicolumn{2}{|c|}{ IV } \\
\hline & \multicolumn{2}{|c|}{40,4} & \multicolumn{2}{|c|}{20,7} & \multicolumn{2}{|c|}{16,2} & \multicolumn{2}{|c|}{22,7} \\
\hline & Moy. & $\sigma$ & Moy. & $\sigma$ & Moy. & $\sigma$ & Moy. & $\sigma$ \\
\hline Un prix de 15 Francs au lieu de 25 Francs & $-1,18$ & 1,17 & 0,20 & 1,43 & 0,66 & 1,24 & 0,57 & 1,21 \\
\hline Sans colorants au lieu de avec colorants et additifs & $\underline{1,48}$ & 1,19 & $-1,31$ & 1,59 & $\underline{1,59}$ & 1,49 & $\underline{1,99}$ & 1,06 \\
\hline L'aspect $100 \%$ naturel des ingrédients & 0,89 & 1,26 & $-0,37$ & 1,60 & $-0,32$ & 1,63 & 0,99 & 1,11 \\
\hline La restitution du vrai goût des ingrédients d'origine & 0,23 & 1,12 & $\underline{2,19}$ & 1,34 & $-0,85$ & 1,63 & $\underline{1,26}$ & 1,04 \\
\hline L'onctuosité (l'aspect crémeux de la glace) & $-0,16$ & 1,07 & $-0,12$ & 1,12 & $\underline{1,52}$ & 1,44 & $-0,63$ & 1,37 \\
\hline Une quantité résiduelle de cristaux d'eau & $-1,26$ & 1,05 & $-0,59$ & 1,53 & $-2,60$ & 1,16 & $-4,18$ & 1,19 \\
\hline Capital marque (en Francs) : & Moy. & $\sigma$ & Moy. & $\sigma$ & Moy. & $\sigma$ & Moy. & $\sigma$ \\
\hline Instants Choisis de MIKO & 0,31 & 5,15 & 0,07 & 5,12 & $\underline{1,31}$ & 4,99 & $-0,01$ & 4,66 \\
\hline Exquise de GERVAIS & $\underline{1,21}$ & 4,90 & 0,82 & 5,30 & 0,56 & 5,10 & 0,02 & 4,46 \\
\hline Carte d'Or de MOTTA & 0,94 & 4,98 & $\underline{2,51}$ & 5,41 & $\underline{1,61}$ & 4,80 & $\underline{2,43}$ & 4,75 \\
\hline CARREFOUR & $-2,45$ & 5,32 & $-3,40$ & 5,75 & $-3,48$ & 5,19 & $-2,44$ & 4,81 \\
\hline
\end{tabular}

Remarquons que le capital marque des 4 marques évaluées (Miko, Gervais, Motta et Carrefour) diffère sensiblement selon les segments. Motta, dont la part de marché est la plus élevée (cf. tableau 2), est aussi la marque la plus appréciée par 3 des 4 segments (soit près de $60 \%$ de l'échantillon) : sa valeur de capital marque est la plus élevée sur les segments II, III et IV. Seule la marque Miko affiche un capital marque comparable, mais uniquement auprès du segment III, tandis que la marque Gervais est la plus fortement valorisée par le segment I. La marque de distributeur Carrefour est également peu appréciée par tous les segments.

Par ailleurs, la valorisation du capital des quatre marques est cohérente avec l'évaluation de l'image de chacune d'entre elles. Ainsi, la différence entre les scores de perception de chaque produit entre les deux phases d'évaluation, en aveugle et avec connaissance de la marque, révèle que la marque Motta est la mieux évaluée sur chaque critère : il n'est donc pas surprenant que cette marque jouisse également du capital marque le plus élevé auprès du plus grand nombre de segments. Autre exemple : Miko, perçue comme une marque onctueuse (diff. $=0,34 ; \mathrm{p}=0,01$ ), séduit davantage le segment III que le segment I : bien que ces deux cibles recherchent toutes deux un produit sans colorant, les consommateurs du segment III se distinguent par l'importance qu'ils attachent à l'onctuosité du produit. En conclusion, il semble exister une relation entre la part de marché d'une marque, sa valeur de capital marque et la perception de son image : cette relation observée au niveau agrégé peut également s'analyser au niveau de chaque segment de marché. 


\section{5)- CONCLUSION}

L'apport de notre recherche que nous plaçons dans un cadre encore exploratoire réside dans une amélioration de la fiabilité et de la validité interne et externe - tout au moins prédictive de la mesure du capital marque proposée par Park et Srinivasan (1994). Notre approche introduit deux perfectionnements. Nous montrons que Park et Srinivasan procèdent à un calcul de différences d'utilités, qui comporte un terme d'erreur propre à la méthode utilisée. Nos résultats expérimentaux attestent que sa valeur est loin d'être négligeable, suggérant ainsi la meilleure validité interne de notre mesure. Certes, à l'instar des deux auteurs, notre mesure individuelle du capital marque suppose que le processus de choix du consommateur peut être décrit par un modèle multiattribut, identique selon que la marque est présente ou occultée (cf. équation 5): certaines marques ont peut-être la capacité à «transformer" le processus de décision du consommateur selon que la marque est connue ou non du répondant. Toutefois, dans le cadre de la mesure que nous proposons, cette hypothèse aboutit à modifier la valeur du capital marque fondée sur les attributs du produit $\left(\mathrm{a}_{\mathrm{ij}}\right)$ sans pour autant remettre en question la quantité capturée par $\mathrm{e}_{\mathrm{ij}}$ (seule la contribution relative des deux composantes $\mathrm{n}_{\mathrm{ij}}$ et $\mathrm{a}_{\mathrm{ij}}$ peut varier).

En outre, nous collectons nos données auprès d'un échantillon unique. Il en résulte deux avantages : un meilleur contrôle des facteurs de distorsion qu'introduit nécessairement le recours à deux échantillons différents et la possibilité de calculer une mesure individuelle (et non agrégée) du capital marque. Certes, le recours à un plan expérimental à mesures répétées n'est pas exempt de critiques. Tout d'abord, il peut provoquer un changement de l'humeur des répondants causé par la fatigue ou l'ennui. Ensuite, il se produit parfois un effet d'interaction, qui se traduit par une plus grande sensibilité des sujets du fait de la mesure préalable. Enfin, il est difficile d'isoler les erreurs de mesure causées par un changement des procédures d'entretien ou du comportement des enquêteurs. Cet aléa est toutefois minoré par le recours à une collecte de données informatisée.

Deux vérifications suggèrent une plus forte validité prédictive de notre méthode révisée. En premier lieu, notre construit de capital marque paraît plus fortement corrélé à d'autres indicateurs de la force de la marque, tels que la part de marché, la distribution numérique et la distribution valeur. Certes, les corrélations observées sont parfois faibles (certaines sont non significatives) et le nombre de marques est ici trop restreint (32) pour prétendre généraliser à ce stade le résultat que nous entrevoyons. La faiblesse surprenant de certaines corrélations est en partie dû aux caractéristiques de marché de certains des produits étudiés : une réplication de la mesure sur un plus grand nombre de catégories de produits est donc souhaitable. Précisons qu'il était difficile pour le répondant de reconnaître la marque des produits : une question préalable de contrôle posée à une partie de l'échantillon a permis de s'assurer de ce point. La présence d'une distorsion purement expérimentale semble donc à écarter.

En second lieu, le modèle de prévision, élaboré à partir de notre méthode, conduit à une estimation satisfaisante des parts de choix : sa qualité d'ajustement aux données, vérifiée au moyen des indicateurs formels de Hauser est bonne. Certes, notre modèle de choix repose sur la prise en compte du dernier achat uniquement : il serait intéressant de réitérer notre mesure en nous appuyant sur des historiques d'achats d'une durée suffisante, issus d'un panel de consommateurs. Bien que cette façon de procéder soit complexe à mettre en œuvre et que les résultats obtenus ne soient pas garantis (Bucklin et Srinivasan, 1991), elle présente, dans le 
contexte de notre recherche, d'indéniables atouts, qui sont autant d'incitations à de futurs travaux. Soulignons enfin que la comparaison entre la part de marché prédite, déclarée et mesurée par un panel ne porte que sur une seule catégorie de produits. La généralisation des résultats supposerait une extension de l'expérimentation à d'autres univers de consommation. Que conclure? Une mesure individuelle du capital marque établie à partir d'une analyse des mesures conjointes est d'un intérêt certain pour le praticien qui souhaite modéliser le comportement de son marché en fonction des évolutions prévisibles de l'offre de produits (Green et Srinivasan, 1990). Notre démarche qui s'inspire des travaux de Park et Srinivasan est une première contribution. Elle porte sur une comparaison essentiellement empirique : établir formellement les aspects fondamentaux de la mesure (notamment sa fiabilité et sa validité convergente et discriminante) suppose au minimum une réplication des résultats sur un plus grand nombre de produits, une procédure de test-retest et le recours à des échantillons de taille plus importante afin de pouvoir utiliser des outils statistiques parfois plus adaptés (équations structurelles, modèles de mélange, etc.). 


\section{ANNEXE 1}

VALEURS DU CAPITAL MARQUE E IJ $_{\mathrm{J}}$ ET MISE EN EVIDENCE DU TERME

D'ERREUR $\xi(\mathrm{O})_{\mathrm{IJ}}$

\begin{tabular}{|c|c|c|c|c|c|}
\hline Marques & $\begin{array}{c}\text { Park et } \\
\text { Srinivasan }\end{array}$ & $\begin{array}{l}\text { Méthode } \\
\text { révisée }\end{array}$ & & $\begin{array}{l}\text { urence ent } \\
\text { ux métho }\end{array}$ & \\
\hline & $\begin{array}{c}\mathrm{e}_{\mathrm{ij}} \\
=\left[\mathrm{u}(\mathrm{s})_{\mathrm{ij}}-\mathrm{v}(\mathrm{o})_{\mathrm{ij}}\right] \\
\text { Moy. }\end{array}$ & $\begin{array}{c}\mathrm{e}_{\mathrm{ij}} \\
=\left[\mathrm{u}(\mathrm{s})_{\mathrm{ij}}-\mathrm{u}(\mathrm{o})_{\mathrm{ij}}\right] \\
\text { Moy. }\end{array}$ & $\begin{array}{l}=[\mathrm{u} \\
\text { Moy. }\end{array}$ & $\begin{array}{l}\left.(\mathrm{o})_{\mathrm{ij}}\right]_{\sigma} \\
\sigma\end{array}$ & $\begin{array}{l}\Omega_{\mathrm{ij}}{ }^{(\mathrm{a})} \\
\text { Moy. }\end{array}$ \\
\hline $\begin{array}{l}\text { Chocolat noir en tablette } \\
\text { Noir de Noir de Côte d'Or } \\
\text { Excellence de Lindt } \\
\text { Grands Chocolats de Nestlé } \\
1848 \text { de Poulain }\end{array}$ & $\begin{array}{r}0,50 \\
0,05 \\
-0,12 \\
-0,43\end{array}$ & $\begin{array}{r}0,06 \\
0,16 \\
-0,01 \\
-0,21\end{array}$ & $\begin{array}{r}0,44 \\
-0,12 \\
-0,11 \\
-0,22\end{array}$ & $\begin{array}{l}4,77 \\
4,93 \\
5,33 \\
4,03\end{array}$ & $\begin{array}{l}1,16 \\
1,03 \\
0,98 \\
0,97\end{array}$ \\
\hline $\begin{array}{l}\text { Champagne brut } \\
\text { Alfred de Rothschild } \\
\text { Veuve Clicquot Ponsardin } \\
\text { Lanson } \\
\text { Taittinger }\end{array}$ & $\begin{array}{r}1,14 \\
4,38 \\
-\quad 7,10 \\
1,57\end{array}$ & $\begin{array}{r}-0,63 \\
5,51 \\
-6,38 \\
1,50\end{array}$ & $\begin{array}{r}1,78 \\
-1,13 \\
-0,72 \\
0,07\end{array}$ & $\begin{array}{l}16,01 \\
15,88 \\
16,58 \\
15,75\end{array}$ & $\begin{array}{l}2,11 \\
2,11 \\
1,05 \\
1,58\end{array}$ \\
\hline $\begin{array}{l}\text { Café soluble } \\
\text { Carte Noire } \\
\text { Maxwell Qualité Filtre } \\
\text { Alta Rica de Nescafé } \\
\text { Nectar de Jacques Vabre }\end{array}$ & $\begin{array}{r}-1,19 \\
5,11 \\
-2,40 \\
-1,51\end{array}$ & $\begin{array}{r}0,35 \\
0,00 \\
-0,15 \\
-0,20\end{array}$ & $\begin{array}{r}-1,54 \\
5,11 \\
-2,25 \\
-1,31\end{array}$ & $\begin{array}{l}4,90 \\
6,62 \\
4,35 \\
4,73\end{array}$ & $\begin{array}{l}0,89 \\
0,99 \\
0,98 \\
0,99\end{array}$ \\
\hline $\begin{array}{l}\text { Crème glacée } \\
\text { Instants Choisis de Miko } \\
\text { Exquise de Gervais } \\
\text { Carte d'Or de Motta } \\
\text { Carrefour }\end{array}$ & $\begin{array}{r}0,39 \\
0,79 \\
1,14 \\
-\quad 2,31\end{array}$ & $\begin{array}{r}0,34 \\
0,69 \\
1,63 \\
-\quad 2,66\end{array}$ & $\begin{array}{r}0,05 \\
0,10 \\
-0,49 \\
0,35\end{array}$ & $\begin{array}{l}4,58 \\
4,20 \\
4,26 \\
3,37\end{array}$ & $\begin{array}{l}0,96 \\
0,99 \\
0,83 \\
0,92\end{array}$ \\
\hline $\begin{array}{l}\text { Carte de crédit bancaire } \\
\text { American Express } \\
\text { Diner's Club } \\
\text { Eurocard / Mastercard } \\
\text { Visa Internationale }\end{array}$ & $\begin{array}{r}53,52 \\
-81,64 \\
-4,57 \\
32,69\end{array}$ & $\begin{array}{r}25,28 \\
-\quad 76,22 \\
1,89 \\
49,06\end{array}$ & $\begin{array}{r}28,24 \\
-5,41 \\
-6,45 \\
-16,37\end{array}$ & $\begin{array}{l}110,47 \\
120,11 \\
124,16 \\
113,71\end{array}$ & $\begin{array}{l}2,60 \\
2,39 \\
2,49 \\
3,05\end{array}$ \\
\hline $\begin{array}{l}\text { Micro-ordinateur } \\
\text { Compaq Presario } \\
\text { Hewlett Packard Pavilion } \\
\text { IBM Aptiva } \\
\text { Packard Bell }\end{array}$ & $\begin{array}{r}235,33 \\
-582,96 \\
691,67 \\
-344,04\end{array}$ & $\begin{array}{r}-150,16 \\
352,70 \\
164,94 \\
-367,48\end{array}$ & $\begin{array}{r}385,49 \\
-935,66 \\
526,73 \\
23,44\end{array}$ & $\begin{array}{r}889,24 \\
1226,61 \\
1066,00 \\
1028,54\end{array}$ & $\begin{array}{l}1,78 \\
3,13 \\
2,21 \\
2,40\end{array}$ \\
\hline $\begin{array}{l}\text { Lave-linge } \\
\text { Arthur Martin } \\
\text { Brandt } \\
\text { Vedette } \\
\text { Whirpool }\end{array}$ & $\begin{array}{r}-74,69 \\
-56,33 \\
394,28 \\
-263,26\end{array}$ & $\begin{array}{r}0,20 \\
-22,33 \\
26,33 \\
-4,20\end{array}$ & $\begin{array}{r}-74,89 \\
-34,00 \\
367,95 \\
-259,06\end{array}$ & $\begin{array}{l}355,53 \\
298,62 \\
260,07 \\
396,69\end{array}$ & $\begin{array}{l}1,76 \\
2,46 \\
1,15 \\
3,09\end{array}$ \\
\hline $\begin{array}{l}\text { Téléviseur } \\
\text { Philips } \\
\text { Radiola } \\
\text { Sony } \\
\text { Thomson }\end{array}$ & $\begin{array}{r}-55,19 \\
-90,57 \\
216,59 \\
-70,83\end{array}$ & $\begin{array}{r}70,14 \\
-178,63 \\
134,99 \\
-26,50\end{array}$ & $\begin{array}{r}-125,33 \\
88,06 \\
81,60 \\
-44,33\end{array}$ & $\begin{array}{l}479,73 \\
404,50 \\
408,42 \\
380,57\end{array}$ & $\begin{array}{l}2,89 \\
2,99 \\
3,43 \\
3,49\end{array}$ \\
\hline
\end{tabular}


ANNEXE 2

VALEURS DU CAPITAL MARQUE, PARTS DE MARCHE, DISTRIBUTION NUMERIQUE ET VALEUR

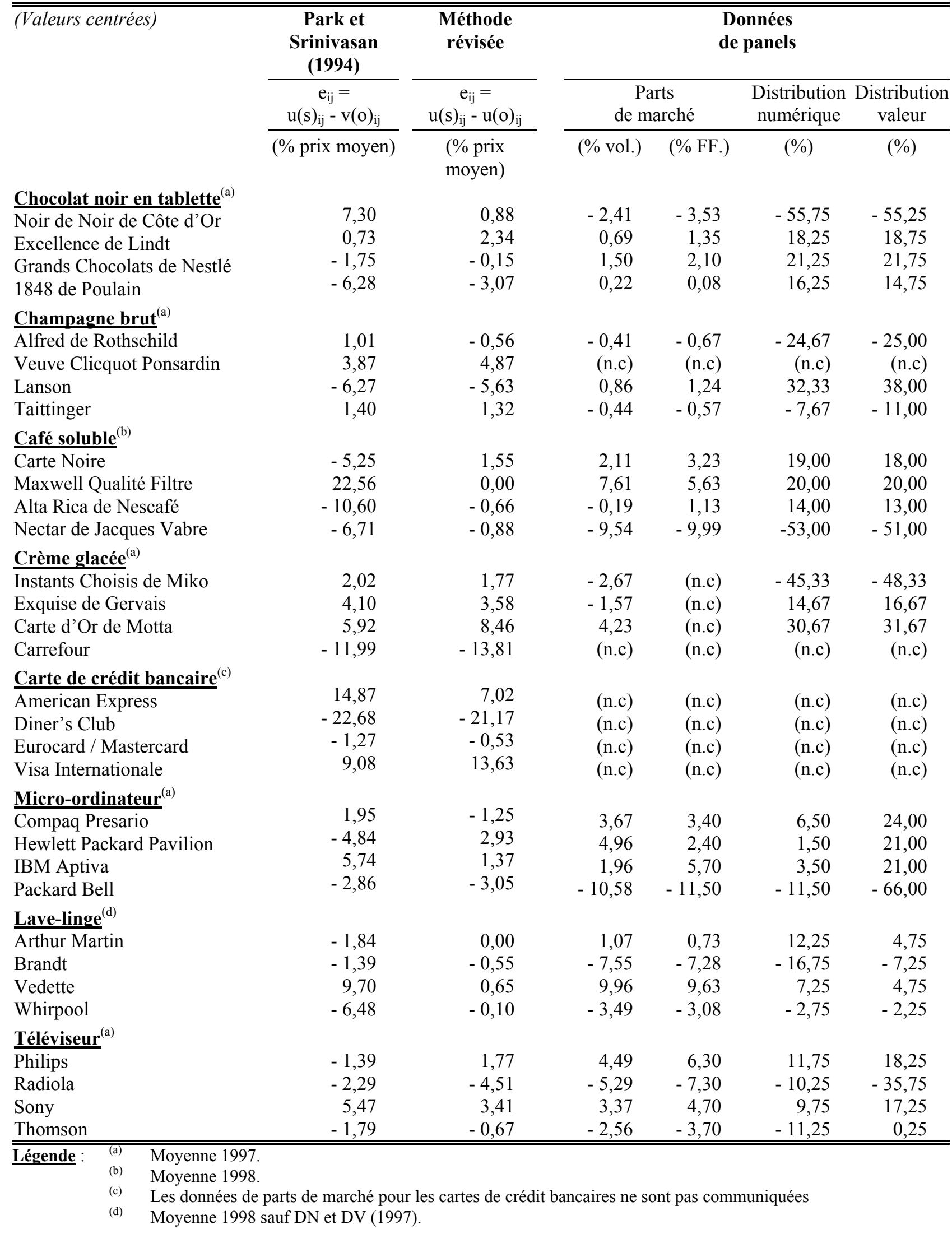




\section{Bibliographie}

Aaker David A. (1991). - Managing Brand Equity: Capitalizing on the Value of a Brand Name. - New York, NY: The Free Press. -299 p.

Aaker David A. (1992). "The Value of Brand Equity”. Journal of Business Strategy, 13, 4, p. 27-32.

Alba Joseph W., Hutchinson John Wesley (1987). “Dimensions of Consumer Expertise'. Journal of Consumer Research, 13, 4, p. 411-454.

Alba Joseph W. , Hutchinson J. Wesley, Lynch John G. Jr. (1991). "Memory and Decision Making,", p. 1-49, in : Handbook of Consumer Behavior/sous la direction de Thomas S. Robertson et Harold H. Kassarjian. -Englewood Cliffs, NJ : Prentice-Hall. 614 p.

Alba Joseph W., Marmorstein Howard (1987). "The Effects of Frequency Knowledge On Consumer Decision Making', Journal of Consumer Research, 14, 1, p. 15-25.

Alpert Mark I. (1971). "Identification of Determinant Attributes : A Comparison of Methods". Journal of Marketing Research, 8, 2, p. 184-191.

Alpert Mark I. (1980). "Unresolved Issues in Identification of Determinant Attributes", p. 83-88, in : Advances in Consumer Research, 7 / sous la direction de Jerry C. Olson (éd.). Ann Arbor, MI : Association for Consumer Research.

Asam Edward H., Bucklin Louis P. (1973). "Nutrition Labeling for Canned Goods : A Study of Consumer Response". Journal of Marketing, 37, 2, p. 32-37.

Barwise Patrick (1993). “Brand Equity : Snark or Boojum ?’. International Journal of Research in Marketing, 10, 1, p. 93-104.

Beckwith Neil E., Lehman Donald R. (1975). “The Importance of Halo Effects in Multi Attributes Models". Journal of Marketing Research, 12, 3, p. 265-275.

Biel Alexander L. (1992). "How Brand Image Drives Brand Equity”. Journal of Advertising, 32, 6, p. 6-12.

Brucks Merrie (1985). "The Effects of Product Class Knowledge on Information Search Behavior'. Journal of Consumer Research, 12, 1, p. 1-16.

Bucklin Randolph E., Srinivasan Seenu V. (1991). "Determining Interbrand Substitutability Through Survey Measurement of Consumer Preference Structures '. Journal of Marketing Research, 28, 1, p. 58-71.

Farquhar Peter H. (1989). “Managing Brand Equity”. Marketing Research, 1, p. 24-33.

Feldman Jack M., Lynch John G. Jr. (1988). "Self-Generated Validity and Other Effects of Measurement on Belief Attitude, Intention and Behavior'. Journal of Applied Psychology, 73, 3, p. 421-435.

Green Paul E., Krieger Abba M., Schaffer Catherine M. (1985). "Quick and Simple Benefit Segmentation'. Journal of Adversiting Research, 25, 3, p. 9-15.

Green Paul E., Srinivasan Seenu V. (1978). "Conjoint Analysis in Consumer Research : Issues and Outlook". Journal of Consumer Research, 5, 2, p. 103-123.

Green Paul E., Srinivasan Seenu V. (1990). "Conjoint Analysis in Marketing : New Developments With Implications for Research and Practice”. Journal of Marketing, 54, 4, p. 3-19.

Hauser John R. (1978). “ Testing the Accuracy, Usefulness, and Significance of Probabilistic Choice Models : An Information-Theoretic Approach.'. Operations Research, 26, 3, p. 406-421.

Hirschman Elisabeth C., Holbrook Morris B. (1982). "Hedonic Consumption : Emerging Concepts, Methods and Propositions'". Journal of Marketing, 46, 3, p. 92-101.

Jacoby Jacob, Chestnut Robert W. (1978). Brand Loyalty : Measurement and Management. New York, NY : John Wiley. 120 p. (Wiley Series on Marketing Management).

Kamakura Wagner A., Russell Gary J. (1993). "Measuring Brand Value with Scanner Data". International Journal of Research in Marketing, 10, Mars, p. 9-22. 
Kapferer Jean-Noël, Thoenig Jean-Claude (1989). La marque : moteur de la compétitivité des entreprises et de la croissance de l'économie. Paris : McGraw Hill. 376 p.

Keller Kevin Lane (1993). "Conceptualizing, Measuring and Managing Customer-Based Brand Equity". Journal of Marketing, 57, 1, p. 1-22.

Krishnan H. Shanker (1996). "Characteristics of Memory Associations : A Consumer-Based Brand Equity Perspective'. International Journal of Research in Marketing, 13, 4, p. 389-405.

Lewi Georges (1996). Sale temps pour les marques. Paris : Albin Michel. 215 p.

Makens J.C. (1965). "Effect of Brand Preference Upon Consumers'Perceived Taste of Turkey Meat'. Journal of Applied Psychology, 49, p. 261-263.

Marketing Science Institute (1988). “Defining, Measuring, and Managing Brand Equity'. Report 88-104 / sous la direction de Lance Leuthesser (éd.). 32 p.

Mazis Michaël B., Ahtola Olli T., Klippel Eugène R. (1975). "A Comparison of Four Multi-Attribute Models in the Prediction of Consumer Attitudes". Journal of Consumer Research, 2, 1, p. 3852.

Murphy Kevin R., Jako Robert A., Anhalt Rebecca L. (1993). "Nature and Consequences of Halo Error : A Critical Analysis". Journal of Applied Psychology, 78, 2, p. 218-225.

Nedungadi Prakash (1990). "Recall and Consumer Consideration Sets : Influencing Choice without Altering Brand Evaluations"'. Journal of Consumer Research, 17, 3, p. 263-276.

Nunnally Jum C. (1978). Psychometric Theory. $2^{\text {ème }}$ éd. New York, NY : McGraw-Hill Book Company. 701 p. (McGraw-Hill series in psychology).

Olson Jerry C. (1972). Cue Utilization in the Quality Perception Process : A Cognitive Model and An Empirical Test. 157 f. dactyl. Thèse de PhD. : Purdue University.

Olson Jerry C. (1977). "Price as an Informational Cue : Effects on Product Evaluations", p. 267-286, in : Consumer and Industrial Buying Behavior / sous la direction de Arch G. Woodside., Jagdish N. Sheth, Peter D. Benett (éd.). New York : North-Holland. 523 p.

Park Chan Su (1992). Estimation and Prediction of Brand Equities Through Survey Measurement of Consumer Preference Structures. 140 f. dactyl. Thèse de PhD. : université de Standford.

Park Chan Su, Srinivasan Seenu V. (1994). "A Survey-Based Method for Measuring and Understanding Brand Equity and Its Extendibility'. Journal of Marketing Research, 31, 2, p. 271-288.

Pessemier Edgar, Burger Philip, Teach Richard, Tigert Douglas (1971). "Using Laboratory Brand Preference Scales to Predict Consumer Brand Purchases'. Management Science, 17, 6, p. 371 385.

Rangaswamy Arvind, Burke Raymond R., Oliva Terence A. (1993). "Brand Equity and the Extendibility of Brand Names". International Journal of Research in Marketing, 10, p. 61-75.

Riezebos H. J. (1994). - Brand-added value : theory and empirical research about the value of brands to consumers. The Netherlands : Eburon Publishers. $-261 \mathrm{p}$.

Silk Alvin J., Urban Glen L. (1978). "Pre-Test Market Evaluation of New Packaged Goods : A Model and Measurement Methodology'. Journal of Marketing Research, 15, 2, p. 171-191.

Shocker Allan D., Weitz Barton A. (1988). "A Perspective on Brand Equity Principles and Issues", in : "Defining, Measuring, and Managing Brand Equity" / sous la direction de Leuthesser Lance (éd.). Report 88-104. Cambridge, MA : Marketing Science Institute. 32 p.

Smith Robert E., Swinyard William R. (1982). “Information Response Model : An Integrated Approach'. Journal of Marketing, 46, 1, p. 81-93.

Srinivasan Seenu V. (1979). "Network Models for Estimating Brand-Specific Effects in MultiAttribute Marketing Models". Management Science, 25, 1, p. 11-21.

Srinivasan Seenu V. (1988). "A Conjunctive-Compensatory Approach to the Self-Explication of Multiattributed Preferences". Decision Science, 19, 2, p. 295-305.

Srivastava Rajendra K., Shocker Allan D. (1991). "Brand Equity : A Perspective on its Meaning and Measurement'. Working Paper, n 91-124. Cambridge, MA : Marketing Science Institute. 
Swait Joffre, Erdem Tulin, Louviere Jordan, Dubelaar Chris (1993). “The Equalization Price : A measure of consumer-perceived brand equity'. International Journal of Research in Marketing, 10,1, p. $23-45$.

Tversky Amos, Kahneman Daniel (1974). "Judgment Under Uncertainty : Heuristic and Biases'. Science, 185, p. 1124-1131.

Tversky Amos, Kahneman Daniel (1998). “Judgment under uncertainty : Heuristics and biases”, p. 3-20, in : Judgment under uncertainty: Heuristics and biases / sous la direction de Daniel Kahneman, Paul Slovic, Amos Tversky (éd.). Cambridge, U.K. : Cambridge University Press. $520 \mathrm{p}$.

Tybout Alice M., Hauser John R. (1981). “A Marketing Audit Using A Conceptual Model of Consumer Behavior : Application and Evaluation”. Journal of Marketing, 45, 3, p. 82-101.

Urban Glen L., Hauser John R. (1980). Design and Marketing of New Products. Englewood Cliffs, NJ : Prentice-Hall. 618 p.

Wilkie William L., Pessemier Edgar A. (1973). "Issues in Marketing Use of Multi-Attribute Attitude Model'”. Journal of Marketing Research, 10, 4, p. 428-441.

Wyer Robert S. Jr., Srull Thomas K. (1986). 'Human Cognition in Its Social Context'” Psychological Review, 93, 3, p. 322-359.

Zajonc Robert B., Markus Hazel (1982). “Affective and Cognitive Factors in Preferences'. Journal of Consumer Research, 9, 2, p. 123-131. 